Draft Version April 12, 2011

Preprint typeset using $\mathrm{L}_{\mathrm{T}} \mathrm{EX}$ style emulateapj v. 11/10/09

\title{
SOLAR MODELS WITH ACCRETION. I. APPLICATION TO THE SOLAR ABUNDANCE PROBLEM
}

\author{
Aldo M. SEREnelli \\ Instituto de Ciencias del Espacio (CSIC-IEEC), Facultad de Ciencias, Campus UAB, 08193 Bellaterra, Spain and \\ Max Planck Institute for Astrophysics, Karl Schwarzschild Str. 1, Garching, D-85471, Germany
}

W. C. HAXTON

Department of Physics, MC 7300, University of California, Berkeley, CA 94720-7300 and Nuclear Science Division, Lawrence Berkeley National Laboratory, Berkeley, CA 94720-8169

AND

Carlos PeÑa-Garay

Instituto de Física Corpuscular (CSIC-UVEG), Aptdo. 22085, E-46071, Valencia, Spain

Draft version April 12, 2011

\begin{abstract}
We generate new standard solar models using newly analyzed nuclear fusion cross sections and present results for helioseismic quantities and solar neutrino fluxes. We discuss the status of the solar abundance problem and investigate whether nonstandard solar models with accretion from the protoplanetary disk might alleviate the problem. We examine a broad range of possibilities, analyzing both metal-enriched and metal-depleted accretion models and exploring three scenarios for the timing of the accretion. Only partial solutions are found: one can bring either the depth of the convective zone or the surface helium abundance into agreement with helioseismic results, but not both simultaneously. In addition, detailed results for solar neutrino fluxes show that neutrinos are a competitive source of information about the solar core and can help constrain possible accretion histories of the Sun. Finally, we briefly discuss how measurements of solar neutrinos from the CN-cycle could shed light on the interaction between the early Sun and its protoplanetary disk.
\end{abstract}

Subject headings: Sun: helioseismology - Sun: interior - Sun: abundances - neutrinos - accretion

\section{INTRODUCTION}

Solar models have been very successful in describing the detailed structure of the solar interior that emerged from helioseismic studies (Christensen-Dalsgaard et al. 1996; Bahcall et al. 2001; Turck-Chièze et al. 2001). Further success came with the first direct measurement of the total ${ }^{8} \mathrm{~B}$ neutrino flux by the SNO Collaboration (Ahmad et al. 2002), which proved to be in excellent agreement with standard solar model (SSM) predictions (Bahcall et al. 2001). Solar models are sensitive to the details of their constitutive physics. Helioseismic predictions, for example, depend critically on radiative opacities and microscopic diffusion, and are also sensitive to uncertainties in input parameters, such as abundances determined from absorption line analyses of the Sun's atmosphere. Predictions of solar neutrino fluxes are sensitive to the rates of key nuclear reactions. Solar models have played a mayor role not only in understanding the Sun, but also as a test bench for constituitive physics of stellar models in general. The further development of SSMs - both improved treatments of the physics and more accurate input parameters - as well as tests of the model against new solar observations are thus of fundamental importance to stellar astrophysics.

Over the last decade, the advent of 3D hydrodynamic models of near-surface solar convection (Stein \& Nordlund 1998; Asplund et al. 2000; Frevtag et al. 2002; Wedemever et al. 2004), a more careful selection of spectral lines (e.g. Allende Prieto et al. 2001) and, in some cases, relaxation of the assumption of local thermodynamic equilibrium in line formation
(Asplund et al. 2004; Bergemann et al. 2010) have resulted in a thorough reassessment of the photospheric solar abundances. The most recent and complete revision by Asplund et al. (2009), hereafter AGSS09, shows a reduction in the abundances of the volatile CNO elements and Ne ( 0.1-0.15 dex) with respect to older compilations of solar abundances (Grevesse \& Noels 1993; Grevesse \& Sauval 1998, hereafter GS98), as well as smaller changes for abundant refractory elements, e.g. Si and Fe. One simple figure-of-merit for comparisons is the photospheric total metal to hydrogen fraction, $(Z / X)_{\odot}$. of $\sim 0.018$ for AGSS09 versus $\sim 0.023$ for GS98. The AGSS09 results, however, have not gone unchallenged. Based on 3D solar atmosphere models computed with the CO5BOLD code (Freytag et al. 2002), Caffau et al. (2010, and references there in) have derived best-value solar CNO abundances that are systematically higher than those in AGSS09 and associated uncertainties that are larger, so that the ranges are formally consistent with GS98 values. The differences between the two groups seem to arise not from the 3D hydrodynamical atmosphere models, but from the selection of spectral lines and assumptions in the line formation calculations.

Nuclear reaction rates are another important input for SSMs. A very broad effort to critically evaluate pp chain and CN cycle rates was undertaken in 1997-8, culminating in Solar Fusion I Adelberger et al. (1998, hereafter SFI). Since SFI a great deal of additional experimental and theoretical work has been done, e.g. ${ }^{14} \mathrm{~N}(\mathrm{p}, \gamma){ }^{15} \mathrm{O}$ (Formicola et al. 2004; Imbriani et al. 2005; Runkle et al. 2005; Bemmerer et al. 2006b; 
Lemut et al. 2006; Marta et al. 2008), ${ }^{3} \mathrm{He}\left({ }^{4} \mathrm{He}, \gamma\right){ }^{7} \mathrm{Be}$ (Singh et al. 2004; Bemmerer et al. 2006a; Gvurky et al. 2007; Confortola et al. 2007; Brown et al. 2007; di Leva et al. 2009), ${ }^{3} \mathrm{He}\left({ }^{3} \mathrm{He}, 2 \mathrm{p}\right){ }^{4} \mathrm{He} \quad$ (Bonetti et al. 1999), and ${ }^{7} \mathrm{Be}(\mathrm{p}, \gamma)^{8} \mathrm{~B}$ (Hammache et al. 1998, 2001; Strieder et al. 2001; Junghans et al. 2002, 2003; Baby et al. 2003a, b; Junghans et al. 2010). The progress includes new facilities for measuring cross sections at low energies in or near the Gamow peak, concerted efforts by independent teams to address key uncertainties such as the S-factor for ${ }^{7} \mathrm{Be}(\mathrm{p}, \gamma)^{8} \mathrm{~B}$, and the development of $a b$ initio methods for theoretical extrapolations to solar energies. In order to assess the impact of this new work on the solar model and other applications, a second evaluation was recently undertaken, establishing new recommended values and uncertainties for pp chain and $\mathrm{CN}$ cycle cross sections (Adelberger et al. 2010, hereafter Solar Fusion II; SFII). Here we present new solar models in which the SFII recommendations are adopted as new standard values, thereby providing the most current best values for SSM neutrino flux predictions and other solar properties.

Standard solar models (SSMs) are calibrated against a present-day surface $(Z / X) \odot$ value: a standard assumption in SSMs is the equivalence between solar core primordial metal abundances and contemporary surface abundances. Early work on SSMs incorporating reduced $\mathrm{CNO}$ abundances (Bahcall et al. 2004; Basu \& Antia 2004; Montalbán et al. 2004) showed that such solar models are typically problematic: the location of the boundary of the convective envelope $\left(R_{\mathrm{CZ}}\right)$, the sound speed and density profiles, and the presentday surface helium abundance $\left(Y_{\mathrm{S}}\right)$ predicted by SSMs using low $(Z / X) \odot$ all differ from the values extracted from helioseismology by amounts beyond accepted uncertainties (Bahcall et al. 2006). On the other hand, SSMs using higher $(Z / X) \odot$ values, e.g. from GS98, show excellent agreement with all helioseismic constraints (Christensen-Dalsgaard et al. 1996; Schlattl et al. 1999; Bahcall et al. 2001, 2005c). The disagreement between 'low-Z' solar model predictions and helioseismic constraints, reminiscent of the now solved 'solar neutrino problem', is popularly known as the 'solar abundance problem'.

Various attempts have been made to solve the solar abundance problem. For example, solar abundance determinations have been questioned (Antia \& Basu 2005, 2006; Bahcall et al. 2005b; Delahaye \& Pinsonneault 2006; Delahave et al. 2010) and the constitutive physics of solar models has been revised (Basu \& Antia 2004; Montalbán et al. 2004; Bahcall et al. 2005a; Guzik \& Mussack 2010). If we assume the correctness of the low-Z metallicity determination for the Sun, there is not, at the moment, a satisfactory solution that brings the solar model into agreement with helioseismology. The only way to achieve agreement in a low$\mathrm{Z}$ model comparable to that achieved with high metallicity (GS98) models is through ad hoc adjustments, such as an increase in opacities to compensate for the lower metal abundance in the solar radiative interior (Christensen-Dalsgaard et al. 2009).

\footnotetext{
1 The equivalence is, of course, modulo the changes produced by microscopic diffusion.
}

An alternative possibility was considered by Guzik (2006), Castro et al. (2007), and more recently by Guzik \& Mussack (2010). These authors assumed that, soon after the Sun settled on the Main Sequence (MS), very metal-poor (or -free) matter was accreted onto the convective envelope, mixing with and diluting its primordial composition. Solar models were constructed with low-metallicity AGSS09-like convective envelopes, but with high-metallicity radiative interiors similar to GS98. It was hypothesized that such a model might have an internal structure consistent with helioseismology, despite its low-metallicity photosphere. However, large discrepancies in model sound speed profiles and convective envelope depths persisted. Additional modifications to the models (extended overshooting, turbulent mixing) were explored, but the difficulties remained, particularly the discrepant sound speed profile below the convective envelope (see Figure 1 in Castro et al. 2007).

While the possibility of solving the solar abundance problem through an inhomogeneous model is intriguing - we know metal inhomogeneities were established late in the evolution of the proto-planetary solar disk because a large excess of metal was sequestered in the planets (Haxton \& Serenelli 2008) - it may be difficult to guess a priori the specific structure such a model should take. The work reported here is a first attempt to examine a broad class of possible nonhomogeneous solar models that could be generated through accretion, which we model dynamically. We explore a range of accretion histories, varying the mass and composition of the gas as well as the epoch during which the accretion occurs. We study the detailed helioseismic properties of these models as well as their neutrino flux characteristics, to determine whether any of the models might be compatible with the various observational constraints. We also consider the implications of the models for future proposed neutrino tests of core composition, such as might be possible through detection of CN-cycle neutrinos (Haxton \& Serenelli 2008) - what classes of accretion models could be directly ruled out by such neutrino measurements? This paper establishes a baseline for future accretion studies that could be more directly tied to recent evidence for peculiar differences in the surface abundances of the Sun and similar stars (solar twins), and possible connections these differences may have to the chemistry of proto-planetary disks (Meléndez et al. 2009). These additional studies will be presented elsewhere.

The paper is laid out as follows. In $\S 2$ we present updated calculations of SSMs for high and low metallicity solar compositions, incorporating the most recent nuclear reaction rates. These models represent the baselines against which models with accretion are later compared. In $\S[$ we describe our calculational procedures: the numerical algorithm and the parameter space the calculations span. Results for accreting solar models are presented in $\S 4$ and discussed in $\S 5$. Final remarks and conclusions are presented in $\S 6$.

\section{STANDARD SOLAR MODELS}

The new SSM calculations presented here were done with GARSTEC (Weiss \& Schlattl 2008), including the updates and modifications in the input physics described in Serenelli et al. (2009). The new models, in addition, 
adopt the nuclear reaction rates that were recently recommended in SFII.

SFII represents the first systematic evaluation of hydrogen burning reactions since the SFI (Adelberger et al. 1998) and NACRE (Angulo et al. 1999) efforts. SFII provides a set of standard S-factors and uncertainties that reflect the progress made in laboratory and theoretical nuclear astrophysics over the last decade. The team of evaluators carefully considered which data sets were sufficiently complete and well documented to be included; how to combine data sets that span different energy ranges and that might be somewhat inconsistent in their error-bar claims; and how to associate reasonable uncertainties to fitting functions (when the data sets span the region of astrophysical interest) or to theoretical extrapolations of data (when no data exist in the solar Gamow peak). The treatment of uncertainties was conservative, due to the increasing relevance of the SSM as a laboratory for weak interaction studies and stellar evolution theory. The principal changes between SFII and SFI are:

$\diamond$ SFI identified ${ }^{7} \mathrm{Be}(\mathrm{p}, \gamma){ }^{8} \mathrm{~B}$ as the least well understood reaction of the pp chain, and called for additional measurements, particularly below $300 \mathrm{keV}$. The SFI evaluation was based on a single data set (Filippone et al. $1983 \mathrm{a}, \mathrm{b})$ due to concern that other results were either too poorly documented or otherwise not sufficiently reliable for inclusion. The SFI recommended S-factor is $\mathrm{S}_{17}(0)=19_{-2}^{+4} \mathrm{eV}$ b. The value used in previous SSM calculations (Serenelli et al. 2009) was $\mathrm{S}_{17}(0)=20.6 \mathrm{eV}$ b (Junghans et al. 2003). The SFII evaluation benefited from four new, high-quality data sets (Hammache et al. 1998, 2001; Strieder et al. 2001; Junghans et al. 2002, 2003; Baby et al. 2003a,b) and yielded $\mathrm{S}_{17}(0)=20.8 \pm 0.7$ (expt) \pm 1.4 (theor). The uncertainty is dominated by the required theoretical extrapolation. The estimated total uncertainty at solar Gamow peak energies is $7.5 \%$.

$\diamond$ The S-factor for the controlling reaction of the CN cycle, ${ }^{14} \mathrm{~N}(\mathrm{p}, \gamma){ }^{15} \mathrm{O}$, was assigned a large and asymmetric uncertainty in SF I, $\mathrm{S}_{114}(0)=3.5_{-1.6}^{+0.4} \mathrm{keV}$ b. A series of new measurements ranging from 70 to $480 \mathrm{keV}$ (Formicola et al. 2004; Imbriani et al. 2005; Runkle et al. 2005; Bemmerer et al. 2006b; Lemut et al. 2006; Marta et al. 2008) has led to a significant reduction in the best value and uncertainty: the SFII result, after summing all transitions, is $\mathrm{S}_{114}(0)=1.66 \pm 0.12 \mathrm{keV}$ b. This value is close to $\mathrm{S}_{114}(0)=1.57 \mathrm{keV}$ b Marta et al. (2008), adopted in our previous SSM results Serenelli et al. (2009). The value and uncertainty are important to future plans to use CN neutrinos as a probe of the solar-core $\mathrm{C}$ and $\mathrm{N}$ abundances, as well as to age estimates for the galaxy's oldest stars.

$\diamond$ Improved measurements were made of the reactions that govern the $\mathrm{ppI} / \mathrm{ppII}+\mathrm{ppIII}$ branching ratio, ${ }^{3} \mathrm{He}\left({ }^{3} \mathrm{He}, \mathrm{pp}\right){ }^{4} \mathrm{He}$ and ${ }^{3} \mathrm{He}\left({ }^{4} \mathrm{He}, \gamma\right){ }^{7} \mathrm{Be}$. The SFI uncertainties for these reactions were $7.4 \%$ and $9.4 \%$, respectively. At the time of SFI, the most recent measurement of the ${ }^{3} \mathrm{He}\left({ }^{4} \mathrm{He}, \gamma\right){ }^{7} \mathrm{Be}$ cross section was ten years old. The new data employed in SFII for ${ }^{3} \mathrm{He}\left({ }^{3} \mathrm{He}, \mathrm{pp}\right){ }^{4} \mathrm{He}$ include measurements that extend to the lower edge of the solar Gamow peak (Bonetti et al. 1999), thus removing any dependence on theory apart from screeening corrections. The new data for ${ }^{3} \mathrm{He}\left({ }^{4} \mathrm{He}, \gamma\right){ }^{7} \mathrm{Be}$ include a series of high-quality measurements (Singh et al. 2004; Bemmerer et al. 2006a; Gyurky et al. 2007; Confortola et al. 2007; Brown et al. 2007; di Leva et al. 2009) at both high and low energies, spanning the range from $93 \mathrm{keV}$ to $2.51 \mathrm{MeV}$. The resulting SFII recommended S-factors are consistent with SFI values but significantly more precise, $\mathrm{S}_{33}(0)=5.21 \pm 0.27 \mathrm{MeV}$ b and $\mathrm{S}_{34}(0)=$ $0.56 \pm 0.03 \mathrm{keV}$ b. The central value of the SFII ratio $\mathrm{S}_{33}(0) / \mathrm{S}_{34}(0)$ is $8.7 \%$ lower than that of $\mathrm{SFI}$, increasing somewhat ppII+ppIII burning. Previously (Serenelli et al. 2009), we had adopted $\mathrm{S}_{34}(0)=0.567$ $\mathrm{keV} b$ Costantini et al. (2008) and $\mathrm{S}_{33}(0)=5.4 \mathrm{MeV}$ b (SFI).

$\diamond$ The S-factor for the driving reaction of the pp chain, $\mathrm{p}\left(\mathrm{p}, \mathrm{e}^{+} \nu_{e}\right) \mathrm{d}$, is taken from theory, as we have no direct means of measuring this reaction currently. The SFI value, $\mathrm{S}_{11}(0)=4.00 \cdot 10^{-22}\left(1 \pm 0.007_{-0.011}^{+0.020}\right) \mathrm{keV}$ $\mathrm{b}$, includes estimated uncertainties from input experimental quantities such as the axial-vector coupling $g_{A}$ and theoretical uncertainties reflecting possible variations in potential-model calculations, including corrections from short-range physics and two-body currents. The most significant development since SFI is the recognition that the overall nuclear physics uncertainty in $\mathrm{S}_{11}$ is governed by a single low-energy parameter which can be constrained by using the precisely know tritium $\beta$ decay rate (Carlson et al. 1991). Consequently three distinct classes of calculations, potential theory (Schiavilla et al. 1998), hybrid effective field theory (EFT) (Park et al. 2003), and pionless EFT (Kong \& Ravendahl 2001; Butler \& Chen 2001; Ando et al. 2008), have now converged in their predictions. The SFII recommended S-factor is $\mathrm{S}_{11}(0)=$ $(4.01 \pm 0.04) \cdot 10^{-22} \mathrm{keV} \mathrm{b}$, and the uncertainty in the Gamow peak is estimated to be $0.9 \%$. The central value is almost $2 \%$ higher than what originally Park et al. (2003) recommended and was used in previous SSM calculations.

$\diamond$ The reaction ${ }^{3} \mathrm{He}\left(\mathrm{p}, \mathrm{e}^{+} \nu_{e}\right){ }^{4} \mathrm{He}$ is the source of the pp chain's most energetic neutrinos. The SFI S-factor best value is $\mathrm{S}_{\text {hep }}(0) \sim 2.3 \cdot 10^{-20} \mathrm{keV}$ b, but the evaluators concluded that no meaningful uncertainty could be assigned. They noted that the scatter of theoretical estimates corresponded to a factor of 2.5, up or down. After SFI there were significant efforts in both potential models (Marcucci et. al. 2000) and hybrid EFT (Park et al. 2003) to improve the calculations and to assess possible sources of uncertainty. The two approaches are in good agreement. The recommended SFII value is $S_{\text {hep }}=(8.6 \pm 2.6) \cdot 10^{-20} \mathrm{keV}$ b.

$\diamond$ New data from the LUNA collaboration on ${ }^{15} \mathrm{~N}(\mathrm{p}, \gamma){ }^{15} \mathrm{O}$ (Bemmerer et al. 2009) that agree with an older data set from Hebbard (1960) but not with Rolfs \& Rodney (1974) led to the SFII conclusion that some unidentified systematic error may have affected the low-energy data of Rolfs \& Rodney 
TABLE 1

MAIN CHARACTERISTICS OF STANDARD SOLAR MODELS.

\begin{tabular}{lcc}
\hline \hline & GS98 & AGSS09 \\
\hline$(Z / X)_{\mathrm{S}}$ & 0.0229 & 0.0178 \\
$Z_{\mathrm{S}}$ & 0.0170 & 0.0134 \\
$Y_{\mathrm{S}}$ & 0.2429 & 0.2319 \\
$R_{\mathrm{CZ}} / R_{\odot}$ & 0.7124 & 0.7231 \\
$\langle\delta c / c\rangle$ & 0.0009 & 0.0037 \\
$Z_{\mathrm{C}}$ & 0.0200 & 0.0159 \\
$Y_{\mathrm{c}}$ & 0.6333 & 0.6222 \\
$Z_{\text {ini }}$ & 0.0187 & 0.0149 \\
$Y_{\text {ini }}$ & 0.2724 & 0.2620
\end{tabular}

(1974). A significantly lower value for $\mathrm{S}_{115}^{\gamma}(0)=36 \pm 6$ $\mathrm{keV} \mathrm{b}$ was recommended as an interim value, pending the outcome of very recent experiments now being analyzed.

$\diamond$ The SFII ratio of pep to pp rates is $2.6 \%$ higher than the corresponding SFI ratio because the former includes a calculation of radiative corrections (Kurvlov et al. 2003) while the later was evaluated at tree level.

These new rates, and others from SFII not explicitly discussed above, were incorporated in the new solar model calculations.

All models in this work are required to reproduce the present-day $\left(\tau_{\odot}=4.57 \mathrm{Gyr}\right)$ solar luminosity, $L_{\odot}=$ $3.8418 \times 10^{33} \mathrm{erg} \mathrm{s}^{-1}$, and radius $R_{\odot}=6.9598 \times 10^{10} \mathrm{~cm}$. In this section we present two SSMs that differ in the present-day surface metal-to-hydrogen abundance ratios. One model is based on the GS98 solar composition, $(Z / X)_{\mathrm{S}}=0.0229$, the other on AGSS09, $(Z / X)_{\mathrm{S}}=$ 0.0178 ; in both cases the adopted scale is meteoritid 2 .

In Table 1 we list the most relevant parameters for these SSMs. The models are labeled according to the adopted solar compositions. The results differ little from those of earlier models with the same respective compositions, presented in Serenelli et al. (2009, Table 2). The SFII reaction rates do not introduce noticeable changes in the initial composition of the models, denoted here by the initial metal, $Z_{\text {ini }}$, and helium, $Y_{\text {ini }}$, mass fractions. Consequently, the surface and central mass fractions of metals, $Z_{\mathrm{S}}$ and $Z_{\mathrm{C}}$, and of helium, $Y_{\mathrm{S}}$ and $Y_{\mathrm{C}}$, are also almost unchanged from those of Serenelli et al. (2009). The depth of the convective envelope $R_{\mathrm{CZ}}$ is slightly deeper in the models with the SFII rates and the average fractional rms deviation between the solar and the model sound speed profiles, $\langle\delta c / c\rangle$, is also slightly better in the new models. The changes are, however, small compared to model uncertainties, so the new models are effectively fully consistent with previous ones.

Given the similarities between the new models and those of Serenelli et al. (2009), the reader is referred to that source for a more detailed discussion. Here, we focus on manifestations of the solar abundance problem, the primary motivation for the studies presented here. There are three key indicators of the abundance problem, the depth of the convective envelope, the surface

\footnotetext{
${ }^{2}$ Except for volatile elements; see Asplund et al. (2009) for details.
}

TABLE 2

SOLAR NEUTRINO FLUXES.

\begin{tabular}{lccc}
\hline \hline$\nu$ flux & GS98 & AGSS09 & Solar $^{\mathrm{a}}$ \\
\hline $\mathrm{pp}$ & $5.98(1 \pm 0.006)$ & $6.03(1 \pm 0.006)$ & $6.05\left(1_{-0.011}^{+0.003}\right)$ \\
pep & $1.44(1 \pm 0.012)$ & $1.47(1 \pm 0.012)$ & $1.46\left(1_{-0.014}^{+0.010}\right)$ \\
hep & $8.04(1 \pm 0.30)$ & $8.31(1 \pm 0.30)$ & $18\left(1_{-0.5}^{+0.4}\right)$ \\
${ }^{7} \mathrm{Be}$ & $5.00(1 \pm 0.07)$ & $4.56(1 \pm 0.07)$ & $4.82\left(1_{-0.04}^{+0.05}\right)$ \\
${ }^{8} \mathrm{~B}$ & $5.58(1 \pm 0.14)$ & $4.59(1 \pm 0.14)$ & $5.00(1 \pm 0.03)$ \\
${ }^{13} \mathrm{~N}$ & $2.96(1 \pm 0.14)$ & $2.17(1 \pm 0.14)$ & $\leq 6.7$ \\
${ }^{15} \mathrm{O}$ & $2.23(1 \pm 0.15)$ & $1.56(1 \pm 0.15)$ & $\leq 3.2$ \\
${ }^{17} \mathrm{~F}$ & $5.52(1 \pm 0.17)$ & $3.40(1 \pm 0.16)$ & $\leq 59$. \\
$\chi^{2} / P^{\text {agr }}$ & $3.5 / 90 \%$ & $3.4 / 90 \%$ & - \\
\end{tabular}

\begin{abstract}
Note. - Neutrino fluxes are given in units of $10^{10}(\mathrm{pp}), 10^{9}\left({ }^{7} \mathrm{Be}\right), 10^{8}\left(\mathrm{pep},{ }^{13} \mathrm{~N},{ }^{15} \mathrm{O}\right), 10^{6}\left({ }^{8} \mathrm{~B},{ }^{17} \mathrm{~F}\right)$ and $10^{3}$ (hep) $\mathrm{cm}^{-2} \mathrm{~s}^{-1}$. Asymmetric uncertainties have been averaged.

a Solar neutrino fluxes inferred from all available neutrino data (Borexino collaboration 2011).
\end{abstract}

helium abundance, and the sound speed profile. The AGSS09 model produces a convective zone that is too shallow and a surface helium abundance that is too low (see Table 11). The entries in the table should be compared to the helioseismic values, $R_{\mathrm{CZ}}=0.713 \pm 0.001$ (Basu \& Antia 1997) and $0.2485 \pm 0.0035$ (Basu \& Antia 2004), respectively. If helioseismic uncertainties are combined with SSM uncertainties (Bahcall et al. 2006, equations 32 and 35), then AGSS09 model predictions differ from solar values at $3 \sigma$ for both $R_{\mathrm{CZ}}$ and $Y_{\mathrm{S}}$. In contrast, GS98 model predictions are in excellent agreement with the parameters extracted from helioseismology. Although more difficult to quantify, the difference between the AGSS09 and solar sound speed profiles, determined from $\langle\delta c / c\rangle$, is about four times larger than that obtained with the higher metallicity GS98 model. These specific discrepancies for the AGSS09 abundance set are the quantitative manifestations of the solar abundance problem mentioned in the introduction.

The new SFII nuclear reaction rates do alter the predicted solar neutrino fluxes, particularly the fluxes associated with the ppIII chain and CN-cycle, mechanisms for solar hydrogen burning that are relatively unimportant energetically. With respect to the rates used in Serenelli et al. (2009), the most important SFII changes are the $2 \%$ increase in $\mathrm{S}_{11}(0)$, the $3 \%$ decrease in $\mathrm{S}_{33}(0)$, and the $5 \%$ increase in $\mathrm{S}_{114}(0)$. Other changes affecting predicted neutrino fluxes include the reduced proton capture cross section on ${ }^{15} \mathrm{~N}$ and the increase in the pep/ pp rate ratio. Model predictions for the neutrino fluxes and associated uncertainties are presented in Table 2 for both the AGSS09 and GS98 compositions. The most significant changes are a $5 \%$ decrease in the predicted ${ }^{8} \mathrm{~B}$ flux primarily because of the increase in $\mathrm{S}_{11}$ and the increase in the ${ }^{13} \mathrm{~N}$ flux due to the larger central abundance of $\mathrm{C}$. The increase in $\mathrm{C}$ is a consequence of the lower SFII value for ${ }^{15} \mathrm{~N}(\mathrm{p}, \gamma){ }^{16} \mathrm{O}$, a reaction that competes with the $\mathrm{CN}$ I cycle reaction ${ }^{15} \mathrm{~N}(\mathrm{p}, \alpha){ }^{12} \mathrm{C}$ and allows mass to flow out of the CN I cycle into CN II. We discuss the parametric dependence of the fluxes on nuclear cross sections and other SSM input parameters elsewhere, including some of the resulting neutrino tests that might be made of solar composition and S-factors (Serenelli et al. 2011). 
Table 2 also includes the updated solar neutrino fluxes inferred from all available neutrino data. The analysis includes the recent more precise, ${ }^{7}$ Be measurement, which is the main change with respect to previous analysis (Bahcall \& Peña-Garav 2003; Peña-Garav \& Serenelli 2008; González-García et al. 2010). Details of the analysis will be shown elsewhere (Borexino collaboration 2011). In order to compare the SSM predictions with the fluxes inferred from neutrino data, we use the $\chi^{2}$ function defined in González-García et al. (2010), with updated errors and correlations. We find $\chi_{\text {GS98 }}^{2}=3.5$ and $\chi_{\mathrm{AGSS} 09}^{2}=3.4$, leading in both cases to $P_{\mathrm{GS} 98, \mathrm{AGSS} 09}^{\mathrm{agr}}=$ $90 \%$. The new fusion cross sections from SFII and the new Borexino results lead to both models predicting solar neutrino fluxes in excellent agreement with inferred ones; from Table 2 it can be seen that solar ${ }^{7} \mathrm{Be}$ and ${ }^{8} \mathrm{~B}$ fluxes are intermediate between SSM predictions for GS98 and AGSS09 compositions. Currently, solar neutrinos can not differentiate between different solar compositions.

\section{SOLAR MODELS WITH ACCRETION: COMPUTATIONAL DETAILS}

\subsection{The algorithm}

Briefly, the construction of an SSM requires finding two parameters that determine the initial composition of the model, $Y_{\text {ini }}$ and $Z_{\text {ini }}\left(X_{\text {ini }}=1-Y_{\text {ini }}-Z_{\text {ini }}\right)$, and the mixing length parameter $\alpha_{\mathrm{MLT}}$. The three parameters are determined iteratively by requiring the model to reproduce the present-day solar luminosity, radius and surface metal-to-hydrogen abundance ratio. We remind the reader that the choice of a solar composition determines not only the present-day $(Z / X)_{\mathrm{S}}$ value, but also the relative abundances of metals within the bulk metallicity. In what follows, all models are computed using the AGSS09 solar composition.

The algorithm used to construct SSMs can be easily modified to compute nonstandard models with accretion, once the properties of the accreted matter (mass and composition) and the timescales and accretion rates are specified. Below, and in the remainder of the paper, we use the following nomenclature: $M_{\mathrm{ac}}$ is the mass of the accreted material, $Z_{\mathrm{ac}}, X_{\mathrm{ac}}$, and $Y_{\mathrm{ac}}$ are, respectively, its metal, hydrogen and helium mass fractions, $\tau_{\text {ac,i }}$ and $\tau_{\text {ac,e }}$ are the initial and ending times of the accretion process. Schematically, the algorithm consists of the following steps:

1. An initial pre-main sequence (PMS) model of mass $M=\mathrm{M}_{\odot}-\mathrm{M}_{\mathrm{ac}}$ is constructed using initial guesses for $Y_{\mathrm{ini}}, Z_{\mathrm{ini}}$, and $\alpha_{\mathrm{MLT}}$.

2. The model is evolved at constant mass until $\tau=$ $\tau_{\text {ac,i }}$. The accretion then begins at a constant rate $\dot{M}$, continuing until $\tau=\tau_{\text {ac,e }}$, when $M=\mathrm{M}_{\odot}$. $Z_{\mathrm{ac}}$ is a parameter of the model, while $X_{\mathrm{ac}}$ and $Y_{\mathrm{ac}}$ are determined by the conditions $X_{\mathrm{ac}} / Y_{\mathrm{ac}}=$ $X_{\text {ini }} / Y_{\text {ini }}$ and $1=X_{\mathrm{ac}}+Y_{\mathrm{ac}}+Z_{\mathrm{ac}}$. That is, we assume that the hydrogen-to-helium ratio of the accreted material is identical to that of the initial solar material.

3. From $\tau=\tau_{\text {ac,e }}$ onward the model is evolved at constant mass until $\tau=\tau_{\odot}$. Model properties are checked against present-day solar values, and the differences are used to improve the initial guesses for $Y_{\mathrm{ini}}, Z_{\mathrm{ini}}$, and $\alpha_{\mathrm{MLT}}$. The process is repeated until the solar surface properties are reproduced to the desired accuracy (typically 1 part in $10^{4}$ or better).

In the above scheme the free parameters of the model are the same as those of SSMs, namely $Y_{\text {ini }}, Z_{\text {ini }}$, and $\alpha_{\mathrm{MLT}}$. But they now depend on properties of the accreted material, particularly its composition, because the mixture of initial and accreted matter that forms the present-day solar surface must satisfy the $(Z / X)_{\mathrm{S}}$ constraint.

So far, we have assumed that the parameters characterizing the accretion process are known. We now discuss how we selected the ranges for those parameters.

\subsection{Models}

The properties of the resulting solar models will clearly depend on the parameters that describe both the properties of the accreted mater and the timing and duration of the accretion process. Our goal was to cover a relatively large parameter space, in order to better define the range of possible solar model outcomes. Below we describe our choices.

\subsubsection{Metallicity of the accreted mass}

Intuitively, a hypothetical solution to the solar abundance problem would seem to require that metal-poor or even metal-free material is accreted onto the Sun. This has been the assumption in the models explored previously (Castro et al. 2007; Guzik \& Mussack 2010). Here we explore metal-free, metal-poor, and metal-rich accretion. Surprisingly, as described in $\S 4$, we find that metalrich accretion can lead to partial improvements in the helioseismic properties of some models. We explore $Z_{\mathrm{ac}}$ values ranging from 0 to 0.03 , the latter being roughly twice the solar surface metallicity. The relative abundances of metals is not varied, but is fixed to AGSS09 values. This is an assumption that may be a reasonable starting point for initial explorations, but probably should be relaxed in future calculations given that any mechanism for segregating metals would likely reflect condensation temperatures and other element-specific chemistry.

\subsubsection{Accreted mass}

We consider accretion of mass from the protoplanetary disk with a composition different from that of the unprocessed material of the proto-solar nebula. The amount of such material the Sun might have accreted in its early evolutionary phases in not well constrained, as estimates of both the mass of the proto-planetary disk and the fraction of that mass that remained in the solar system vary. Assuming that initially the proto-solar cloud was well mixed, one can set a lower limit to the mass of the protoplanetary disk, the so-called minimum-mass solar nebula (MMSN) limit, from estimates of the metal content of the planets. The calculation, however, still depends on model assumptions. Early estimates (Weidenschilling 1977; Havashi 1981) give $M_{\mathrm{MMSN}} \approx 0.013-0.07 \mathrm{M}_{\odot}$, assuming planets were formed at their present locations. More sophisticated models that take into account migration give somewhat larger values. Desch (2007) finds 
$M_{\mathrm{MMSN}} \approx 0.17 \mathrm{M}_{\odot}$ but with a disk that is being strongly photo-evaporated, i.e., most of its mass migrates outwards, not towards the Sun.

Based on these considerations, we explore values of $M_{\mathrm{ac}}$ in the range from $3 \cdot 10^{-5}$ to $0.06 \mathrm{M}_{\odot}$, or equivalently, from 10 to $2 \cdot 10^{4} \mathrm{M}_{\oplus}$. We stress that this is the total accreted mass, not the metal mass. The upper end of the range thus comes within a factor of 2-3 of most estimates of $M_{\mathrm{MMSN}}$. Given that models of massive disks with steep density profiles lead to substantial (Desch 2007) evaporation, $0.06 \mathrm{M}_{\odot}$ is probably a safe upper bound on the amount of mass that could have been chemically processed in the protoplanetary disk and later accreted onto the Sun. In terms of the total accreted metal, our choices for $M_{\mathrm{ac}}$ and $Z_{\mathrm{ac}}$ yield a range of 0 to $600 \mathrm{M}_{\oplus}$.

\subsubsection{Accretion timescales and mass accretion rate}

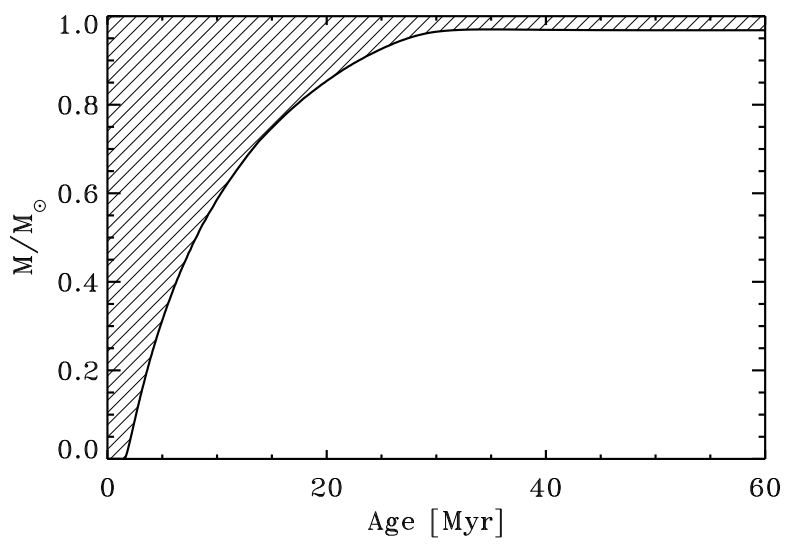

Fig. 1.- Time dependence of $M_{\mathrm{CE}}$ during early evolutionary phases of a solar model; the shaded area depicts the convective zone. $M / \mathrm{M}_{\odot}=1$ defines the surface of the model.

The time when accretion takes place is of particular importance. This is clear from inspection of Figure1, where the evolution of the mass of the convective envelope, $M_{\mathrm{CE}}$, during early $\mathrm{SSM}$ evolution is shown. Initially, the model is fully convective on the Hayashi track. As it evolves towards the Zero Age Main Sequence (ZAMS), a radiative core develops and convection quickly retreats. During these early phases, the $e$-folding time for the evolution of $M_{\mathrm{CE}}$ is about $8 \mathrm{Myr}$. At about $30 \mathrm{Myr}$, the star reaches the ZAMS and further evolutionary changes, including the extension of the convective envelope, occur on very long (nuclear) timescales. For a fixed amount of accreted material, the mass of the convective envelope at the time of accretion determines the degree of dilution (or enrichment). These considerations lead us to identify three paradigmatic accretion scenarios:

$\diamond$ early accretion: Accretion starts when the convective envelope still encompasses most of the star; accreted material is then highly diluted. We take $\tau_{\mathrm{ac}, \mathrm{i}}=5 \mathrm{Myr}$ as a representative time for the early accretion scenario. In typical models, the convective envelope contains 70 to $80 \%$ of the total solar mass at this time.

$\diamond$ intermediate accretion: Accretion starts when the convective envelope has receded towards the surface and contains about 20 to $30 \%$ of the stellar mass. Accreted material is strongly diluted in the envelope but the effects of accretion in the final composition of the model are clearly noticeable. We take $\tau_{\mathrm{ac}, \mathrm{i}}=15 \mathrm{Myr}$ as representative of this case;

$\diamond$ late accretion: Accretion starts when the model is very close to the ZAMS, i.e. when timescales for structural changes begin to be dominated by nuclear burning timescales. The convective envelope is mature, close to its final MS size, and changes very little afterwards. Its mass is comparable to some of the largest $M_{\mathrm{ac}}$ values we consider. Accordingly, the final structure of models with late accretion is strongly affected by the properties of the accreted material. We take $\tau_{\mathrm{ac}, \mathrm{i}}=30 \mathrm{Myr}$ as representative of late accretion.

Accretion could occur at even later times. However, given that $M_{\mathrm{CE}}$ changes very little after the first $30 \mathrm{Myr}$ of evolution, such models should not differ significantly from the late accretion scenario described above. In terms of involved timescales, the late accretion scenario is equivalent to that investigated by Castro et al. (2007) and Guzik \& Mussack (2010), who assumed accretion took place once the Sun had settled on the MS.

Computationally, the accretion scenarios are defined in terms of the time at which accretion takes place. Physically, however, the relevant characteristic is the thickness of the convective envelope. Therefore, as long as accretion occurs sufficiently early that element diffusion effects are still negligible, the thickness of the convective envelope is the only physically relevant parameter. For this reason, even if the young Sun avoided a fully convective Hayashi phase (Wuchterl \& Klessen 2001; Baraffe \& Chabrier 2010) and consequently developed a thin convective envelope after only a few $\mathrm{Myr}$ (Baraffe \& Chabrier 2010), our intermediate and late accretion results remain valid, constraining these alternative evolutionary tracks.

The duration of the accretion phase $\left(\Delta \tau_{\mathrm{ac}}\right)$ is a second important parameter, particularly for the early and intermediate cases, where $M_{\mathrm{CE}}$ evolves rapidly. Our standard choice is $\Delta \tau_{\mathrm{ac}}=10 \mathrm{Myr}$. There are two reasons for this choice. One is simplicity: this choice corresponds to quiescent accretion rates well below $10^{-7} \mathrm{M}_{\odot} \mathrm{yr}^{-1}$, allowing us to neglect the energy deposition on the outer layers of the star and associated effects that could alter final internal structure of the model (Baraffe et al. 2009). We assume that the central star is almost completely assembled when accretion from the protoplanetary disk, that is accretion of chemically processed material, begins. The second reason is the correspondence to typical estimates of the planetary formation time, usually in the range of a few to 10 Myr. However, to test the impact of different accretion durations, we have computed models with more rapid accretion, $\Delta \tau_{\mathrm{ac}}=1 \mathrm{Myr}$.

In all cases considered, accretion is assumed to occur at a constant mass rate $\dot{M}=M_{\mathrm{ac}} / \Delta \tau_{\mathrm{ac}}$.

\subsection{Grid of models}

The ranges considered for $M_{\mathrm{ac}}$ and $Z_{\mathrm{ac}}$ were explored by stepping through a $10 \times 10$ grid, with quadratic spacings. Therefore, for each value of $\tau_{\mathrm{ac}, \mathrm{i}}$ and $\Delta \tau_{\mathrm{ac}}, 100$ models were generated. This basic grid is depicted in Figure 2. 


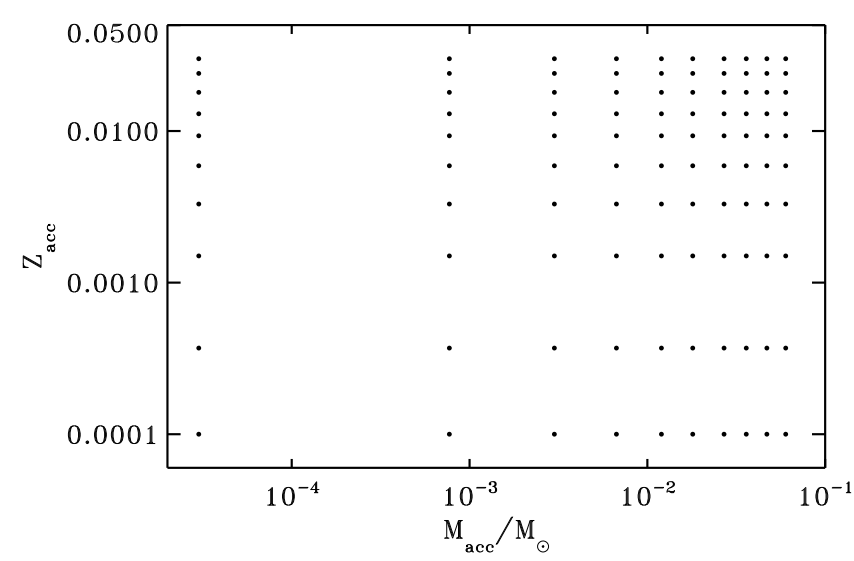

FIG. 2.- The mass and metallicity grid for the model calculations.

\section{SOLAR MODELS WITH ACCRETION: RESULTS}

Here we present results for the three accretion scenarios discussed above, early, intermediate, and late. We first focus on the relationship between the altered solar composition and helioseismic observables, then later discuss the effects on neutrino fluxes.

\subsection{Early accretion}

\subsubsection{Composition and helioseismic quantities}

Model compositions and resulting helioseismic quantities are shown in Figure 3 for five selected model sequences of constant $Z_{\mathrm{ac}}$, from metal-free to super-solar metallicity. Panels $a$ and $b$ give the initial composition of the models, $Y_{\text {ini }}$ and $Z_{\text {ini }}$ respectively, i.e. the composition before accretion takes place. Panel $c$ shows the present-day surface metallicity $Z_{\mathrm{S}}$. The lower panels show selected solar properties that can be directly tested with helioseismology, $R_{\mathrm{CZ}}$ in panel $d,\langle\delta c / c\rangle$ in panel $e$, and $Y_{\mathrm{S}}$ in panel $f$.

We first examine the behavior of the initial composition of the models. Since all models have to satisfy $(Z / X)_{\mathrm{S}}=0.0178$, metal-poor accretion models lead to higher $Z_{\text {ini }}$ values. A higher metallicity core implies an increase in the radiative opacity and molecular weight, i.e., larger central temperatures. However, models are constrained by $\mathrm{L}_{\odot}$ as well. So, an increase in nuclear burning caused by higher central temperatures has to be compensated by limiting the amount of fuel available,

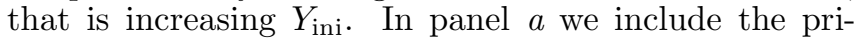
mordial helium mass fraction from Standard Big Bang Nucleosynthesis $(\mathrm{SBBN}), Y_{\mathrm{P}}=0.2486 \pm 0.0006$, using the latest WMAP results for the baryon-to-photon rato $\eta$ (Larson et al. 2010).

The effects of accretion on metallicity profiles are shown in Figure 4 for three models with $M_{\mathrm{ac}}=0.06$. The models are identified in panel $e$ by the open circles. For comparison, we included the results for the SSMs presented in $\S 2$. The strong gradient in the metallicity profile between $\sim 0.17$ and $0.35 \mathrm{R}_{\odot}$ seen for the metalrich and metal-free models is the result of mixing between the accreted and the primordial material, while the convective zone is receding towards the surface. As discussed above, the surface metallicity is very similar in all models and nearly equal to that of the AGSS09 SSM.
Model values for $R_{\mathrm{CZ}},\langle\delta c / c\rangle$, and $Y_{\mathrm{S}}$, quantities tightly constrained by helioseismology, are shown in panels $d, e$, and $f$ of Figure 3. respectively. Panels $d$ and $f$ also include the helioseismic values for $R_{\mathrm{CZ}}$ and $Y_{\mathrm{S}}$, plotted as horizontal solid lines, and their $1 \sigma$ uncertainties, shown as dotted lines. We first discuss the behavior of $R_{\mathrm{CZ}}$ as a function of $Z_{\mathrm{ac}}$. The outer layers (above $0.8 \mathrm{M}_{\odot}$, see Figure 4) of models evolved with metal-poor accretion have lower metallicities. Additionally, lower $Z_{\mathrm{S}}$ requires lower $X_{\mathrm{S}}$. Both effects tend to lower the radiative opacity at $R_{\mathrm{CZ}}$ (Bahcall et al. 2004), producing a shallower convective envelope. The opposite is true for metal-rich accretion. Therefore, the behavior of $R_{\mathrm{CZ}}$ seems to argue against metal-free (or metal-poor) accretion. In contrast, metal-rich accretion leads to deeper convective envelopes, closer to the helioseismic value. The trends in the behavior of the sound speed profiles are quite similar, as one sees from panel $e$. There the GS98 SSM result is shown as a horizontal line. The behavior of $\langle\delta c / c\rangle$ and $R_{\mathrm{CZ}}$ are very well correlated because the bulk of the discrepancy in the sound speed profile comes from the mismatch between the solar and model $R_{\mathrm{CZ}}$ values. To show this, we plot in Figure 5 the relative sound speed difference as a function of radius for the same models shown in Figure 4 and identified in panel $e$ with open circles. The bump below the convective zone decreases for models with $R_{\mathrm{CZ}}$ close to the solar value (vertical dotted line). This occurs for metal-rich accretion models. The SSM results for AGSS09 and GS98 compositions are included for comparison.

Unfortunately, the behavior of $Y_{\mathrm{S}}$ is qualitatively opposite that of $R_{\mathrm{CZ}}$ and $\langle\delta c / c\rangle$. Metal-poor accretion leads to higher $Y_{\mathrm{S}}$ values as a result of larger $Y_{\text {ini }}$ (Serenelli \& Basu 2010), improving the agreement with the helioseismic determination. In contrast, metal-rich accretion leads to lower, more discrepant, $Y_{\mathrm{S}}$ values. Therefore, helium abundance considerations favor metalpoor accretion.

These results suggest that it may not be possible to construct solar models with early accretion in which all predicted helioseismic variables agree with solar values extracted from helioseismic observations. While agreement for $R_{\mathrm{CZ}}$ and $\langle\delta c / c\rangle$ can be improved with metalrich accretion, the accompanying lower $Y_{\mathrm{S}}$ values increase discrepancies for that variable. Similarly, metal-poor accretion helps with $Y_{\mathrm{S}}$, but increases the discrepancies in $R_{\mathrm{CZ}}$ and $\langle\delta c / c\rangle$.

\subsubsection{Neutrino fluxes}

Solar neutrino fluxes place a second set of constraints on solar interior properties. We will compare model predictions to the flux values determined from a global analysis of solar neutrino experiments (Borexino collaboration 2011) that are given in the last column of Table 2, The authors considered all available data from solar neutrino experiments, including new determinations of the ${ }^{8} \mathrm{~B}$ flux by SNO (Aharmim et al. 2010) and Borexino (Bellini et al. 2010) and the most recent measurement of the ${ }^{7} \mathrm{Be}$ flux by Borexino Arpesella et al. 2008; Borexino collaboration 2011).

The general dependence of solar model fluxes on accretion simply reflects the initial metallicity, which determines the temperature in the Sun's energy-producing 

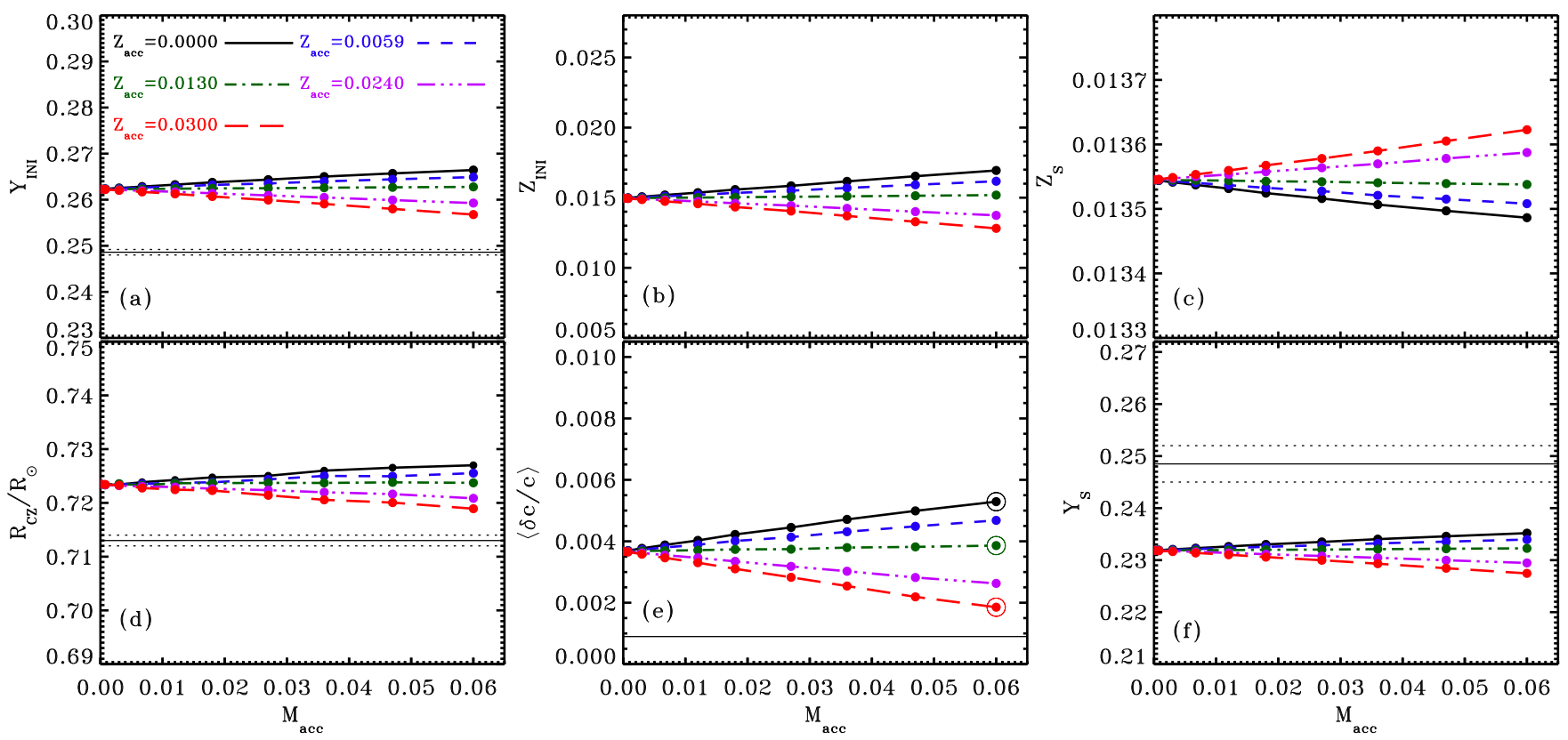

FIG. 3.- Composition and helioseismic quantities of sequences of accreting solar models with $\tau_{\mathrm{ac}, \mathrm{i}}=5 \mathrm{Myr}$ and $\Delta \tau_{\mathrm{ac}}=10 \mathrm{Myr}(\mathrm{early}$ accretion). Results are shown for five different values of $Z_{\mathrm{ac}}$, as labeled in panel $a$.

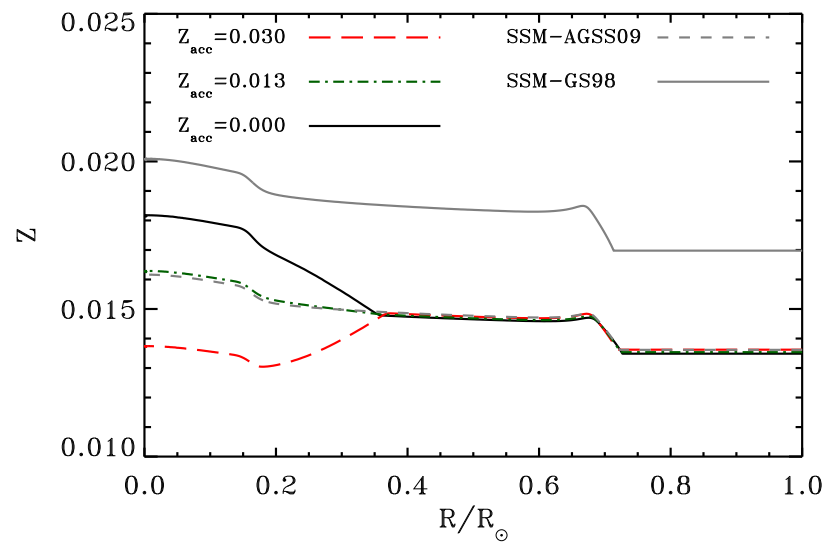

FiG. 4. - Final metallicity profile as a function of radius, for three accreting models with the $Z_{\text {ac }}$ values indicated in the plot and for $M_{\mathrm{ac}}=0.06 \mathrm{M}_{\odot}$. For comparison, the SSM metallicity profiles are also shown (

core. Fluxes that depend more strongly on temperature $\left({ }^{8} \mathrm{~B},{ }^{13} \mathrm{~N},{ }^{15} \mathrm{O},{ }^{17} \mathrm{~F}\right.$ and, to a lesser extent, $\left.{ }^{7} \mathrm{Be}\right)$ increase with increasing core metallicity (metal-poor accretion). However, because the models are constrained by $L=L_{\odot}$, neutrino fluxes less dependent on core temperature (pp, pep and hep) must decrease (Bahcall \& Ulmer 1996). In our accretion models the dependence of each neutrino flux on solar core metallicity can be characterized by the partial derivatives

$$
\frac{\partial \log \Phi\left(\nu_{i}\right)}{\partial \log Z_{\text {ini }}}=\alpha\left(\nu_{i}\right) .
$$

We determine $\alpha\left(\nu_{i}\right)$, for each flux $i$, by performing a joint linear fit to all early accretion models. The fluxes most strongly correlated with core temperature yield $\alpha\left({ }^{7} \mathrm{Be}\right)=$ $0.34, \alpha\left({ }^{8} \mathrm{~B}\right)=0.82, \alpha\left({ }^{13} \mathrm{~N}+{ }^{15} \mathrm{O}\right)=1.58$. For pp and pep fluxes we find $\alpha(\mathrm{pp})=-0.036, \alpha(\mathrm{pep})=-0.16$. In what

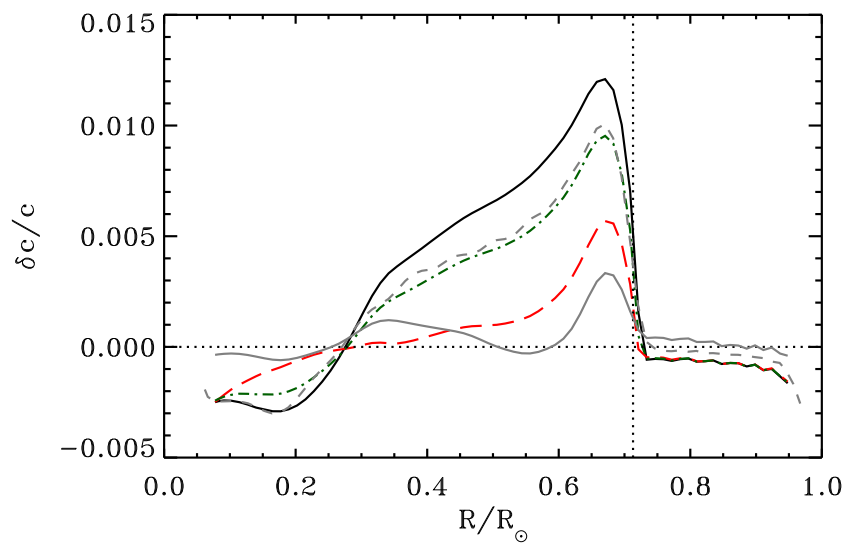

Fig. 5.- Sound speed profiles for the models of Figure 4 The vertical dotted line marks the boundary of the convective envelope.

follows, the sum of the fluxes ${ }^{13} \mathrm{~N}+{ }^{15} \mathrm{O}$ will be denoted CN.

Results for the various neutrino fluxes are shown in Figure 6. The metallicity sequences are the same as in Figure 3 . Panels $a$ and $b$ show the ${ }^{8} \mathrm{~B}$ and ${ }^{7} \mathrm{Be}$ fluxes, which have been measured directly in experiments (the ${ }^{7}$ Be flux can be extracted from Borexino results given other measurements of neutrino oscillation parameters). The solar fluxes (third column, Table 2) are shown with horizontal black lines, including $1 \sigma$ uncertainties. Models with metal-rich accretion tend to deviate from the solar values, particularly for the precisely known ${ }^{8} \mathrm{~B}$ flux due to its sensitivity to core temperature. As mentioned before, the greatest sensitivity to accretion is exhibited by the combined CN flux (panel $c$ ) because, in addition to its dependence on core temperature, this flux depends linearly on the total $\mathrm{C}+\mathrm{N}$ abundance in the solar core. Unfortunately, the current determination of the combined flux is only an upper limit, $\Phi(\mathrm{CN}) \leq 9.9 \times 10^{8} \mathrm{~cm}^{-2} \mathrm{~s}^{-1}$, and 

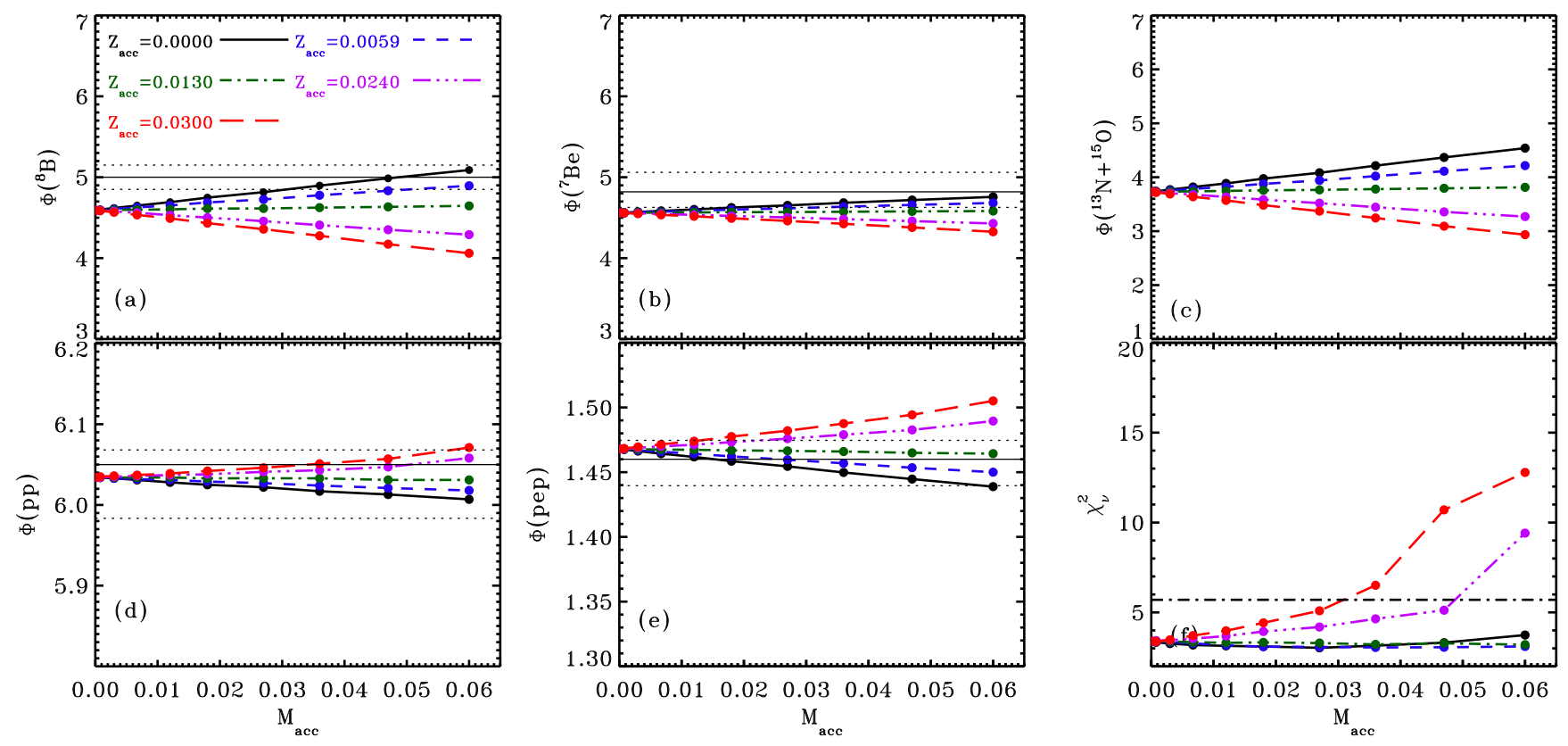

FIG. 6.- Neutrino fluxes for the accreting solar models of Figure 3
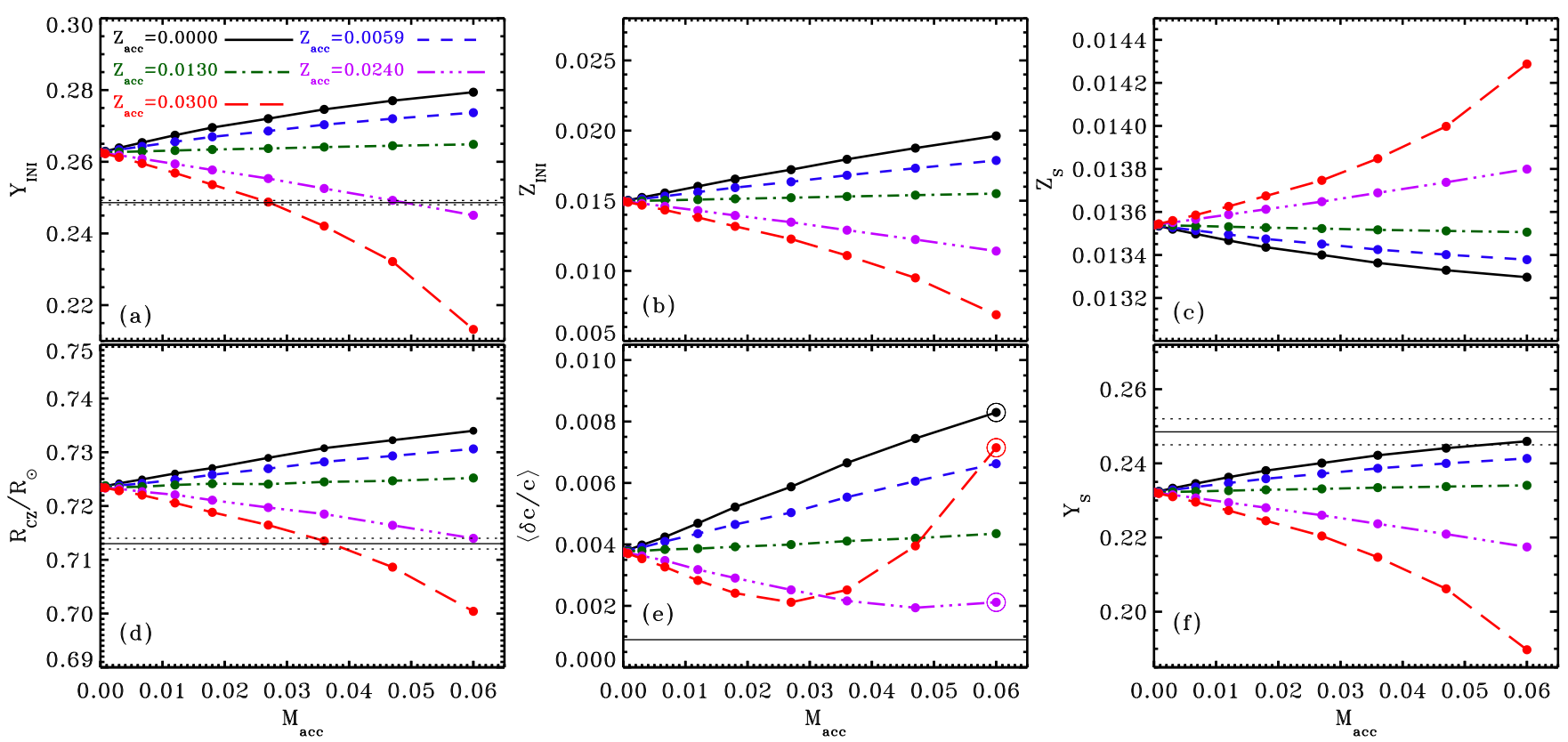

FIG. 7.- Composition and helioseismic quantities of sequences of accreting solar models with $\tau_{\mathrm{ac}, \mathrm{i}}=15 \mathrm{Myr}$ and $\Delta \tau_{\mathrm{ac}}=10 \mathrm{Myr}$ (intermediate accretion). Results are shown for five different values of $Z_{\mathrm{ac}}$, as labeled in panel $a$.

does not allow to put any constraints on the solar core metallicity.

Panels $d$ and $e$ show the strongly correlated pp and pep fluxes. The luminosity constraint and the ${ }^{7} \mathrm{Be}$ measurement by Borexino combine to produce the small uncertainties in these fluxes (about $1 \%$ and $1.5 \%$, respectively). As with $\Phi\left({ }^{8} \mathrm{~B}\right)$, particularly the pep flux somewhat disfavors metal-rich accretion models, especially those with large $M_{\text {ac }}$ values.

As an indicator of the overall agreement between model and solar fluxes, we calculated, for each model, the $\chi^{2}$ function (see $\S 2$ ). The results are shown in panel $f$. The horizontal dashed-dotted line shows the $68.3 \%$ probability band. As expected from results for individual fluxes, metal-poor accretion models lead to a small overall improvement in the agreement with experimental fluxes, mostly driven by improvements in the agreement with $\Phi\left({ }^{8} \mathrm{~B}\right)$ and $\Phi(\mathrm{pep})$. Note that the sequence with $Z_{\mathrm{ac}}=0.0130$ shows almost no changes in the predicted neutrino fluxes and $\chi^{2}$ values: this value of $Z_{\mathrm{ac}}$ is nearly equal to the AGSS09 $Z_{\text {ini }}$ value, so that the accreted material and initial solar material have nearly identical compositions. We caution that these neutrino flux changes should not be overinterpretted, as we have seen that models with metal-poor accretion tend to do 
poorly on $R_{\mathrm{CZ}}$ and $\langle\delta c / c\rangle$ and therefore their internal structure shows differences with respect to the Sun.

\subsection{Intermediate accretion}

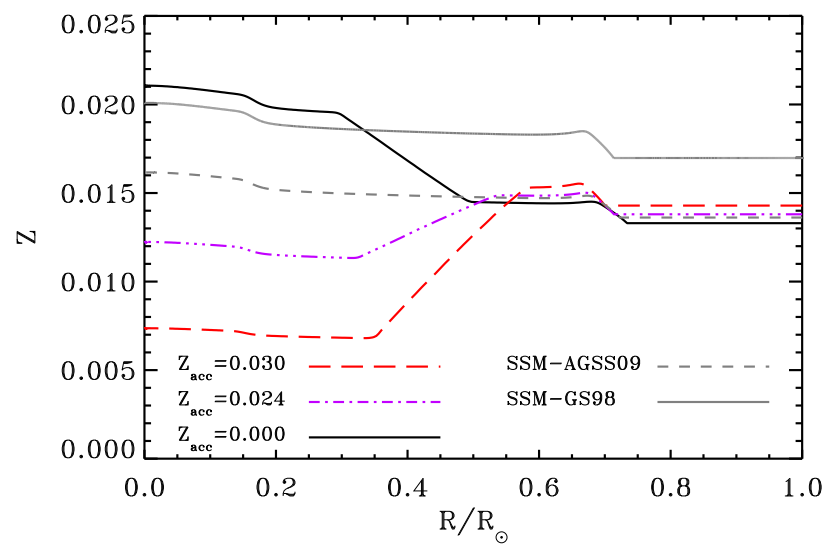

FIG. 8.- Metallicity profiles for selected models (open circles in panel $e$ in Figure 7). SSM results are also shown, as in Figure 4

\subsubsection{Composition and helioseismic quantities}

The results for $Y_{\mathrm{ini}}, Z_{\mathrm{ini}}$, and $Z_{\mathrm{S}}$ are shown in panels $a, b$, and $c$, respectively, of Figure 7. The qualitative trends track those we observed in early accretion models: metal-poor accretion leads to higher $Z_{\text {ini }}$ and $Y_{\text {ini }}$ and, because of the lower $X_{\text {ini }}$, lower $Z_{\mathrm{S}}$. Metal-rich accretion produces the opposite behavior. Metal-rich accretion for $Z_{\mathrm{ac}}=0.024$ and 0.030 and with accreted masses above 0.05 and $0.027 \mathrm{M}_{\odot}$, respectively, lead to $Y_{\text {ini }}$ lower than the SBBN primordial value $Y_{\mathrm{P}}$. The same models lead to $Z_{\text {ini }}$ values that are low when compared to metallicities observed in the solar neighborhood (see e.g. Przybilla et al. 2008). Compared to early accretion models, the consequences of accretion are amplified in the intermediate models because the convective envelopes are shallower at the time of accretion. The large contrast between $Z_{\text {ini }}$ and $Z_{\mathrm{S}}$ for intermediate accretion models is apparent from Figure 8, where the metallicity profiles for the three models identified with open circles in Figure 7 are shown. The partially mixed region extends roughly between 0.35 and $0.55 \mathrm{R}_{\odot}$, depending on the model. Profiles of the GS98 and AGSS09 SSMs are also shown. Metal-rich accretion models, as stated above, lead to under-metallic cores. The consequences for resulting model sound-speed profiles are significant, as discussed below.

Results for the helioseismic quantities $R_{\mathrm{CZ}},\langle\delta c / c\rangle$, and $Y_{\mathrm{S}}$ are shown in panels $d, e$, and $f$ of Figure 7 respectively. The qualitative behavior of $R_{\mathrm{CZ}}$ and $Y_{\mathrm{S}}$ is quite similar to that found in the early accretion models. Interestingly, models with metal-rich accretion can lead to $R_{\mathrm{CZ}}$ in excellent agreement with the helioseismic value. However, the same models yield $Y_{\mathrm{S}}$ values that are low compared to helioseismic values, as well as $Y_{\text {ini }}$ values below the SBBN value. For $Z_{\mathrm{ac}}=0.030$, the highest $M_{\mathrm{ac}}$ values considered, the convective envelopes are deeper than allowed by helioseismology. This is reflected in the behavior of $\langle\delta c / c\rangle$. For the $Z_{\mathrm{ac}}=0.03$ sequence, $\langle\delta c / c\rangle$

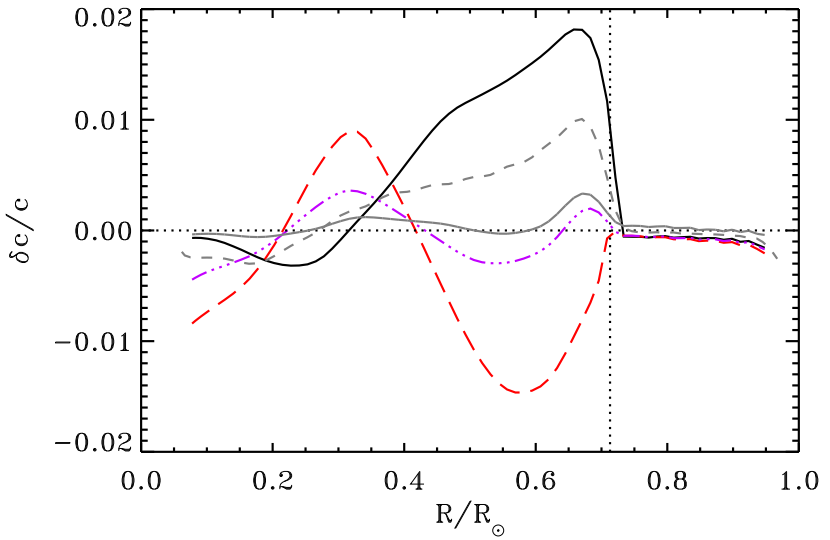

FIG. 9.- Sound speed profiles for the models of Figure 8

has a minimum around $M_{\mathrm{ac}}=0.027 \mathrm{M}_{\odot}$. The agreement is better than with the AGSS09 SSM, but still a factor of two worse than with the GS98 SSM. The agreement deteriorates for larger $M_{\mathrm{ac}}$ values, as the convective envelope becomes too deep. Figure 9 gives the sound speed profiles for the same models that were used in Figure 8. The sound speed profile for the model with metal-free accretion (black solid line) is significantly degraded compared to that of AGSS09 SSM, because the convective envelope is too shallow; the bump below the convective zone is very large and extends quite deep, to $\sim 0.35 \mathrm{R}_{\odot}$. The difficulty exists despite the similarity between the metallicity of this model and that of AGSS09 SSM over a substantial region, $0.45-0.7 \mathrm{R}_{\odot}$, below their convective envelopes (Figure 8 for $M>0.9 \mathrm{M}_{\odot}$ ). For $Z_{\mathrm{ac}}=0.024$ and $M_{\mathrm{ac}}=0.06 \mathrm{M}_{\odot}$ (green line-dotted line), the model predicts the correct $R_{\mathrm{CZ}}$, so that the bump below the convective envelope is almost gone. At small $r$, the sound speed difference oscillates around zero, and the overall $\langle\delta c / c\rangle$ is twice as large as for the GS98 SSM (shown as the grey dashed line). Finally, the model with $Z_{\text {ac }}=0.030$, which we noted had too deep a convective zone, exhibits a large bump in the sound speed profile of opposite sign below the convective zone, as well as significant discrepancies at smaller radii due to its under-metallic interior.

\subsubsection{Neutrino fluxes}

Relative to early accretion models, the neutrino fluxes from intermediate accretion models exhibit slightly more sensitivity to $Z_{\mathrm{ini}}: \quad \alpha\left({ }^{7} \mathrm{Be}\right)=0.52, \alpha\left({ }^{8} \mathrm{~B}\right)=1.12$, $\alpha(\mathrm{CN})=1.77, \alpha(\mathrm{pp})=-0.045$, and $\alpha(\mathrm{pep})=-0.14$. Flux results are shown in Figure 10. The higher sensitivity of temperature-dependent fluxes, particularly $\Phi\left({ }^{8} \mathrm{~B}\right)$, $\Phi\left({ }^{7} \mathrm{Be}\right)$, and the combined $\Phi(\mathrm{CN})$, reflects the decreased dilution of accreted material. Panel $a$ shows that $\Phi\left({ }^{8} \mathrm{~B}\right)$, in models with metal-rich accretion, differs markedly from the solar value (horizontal black line). Conversely, metal-poor accretion modestly improves the agreement with experiment for almost all the range of $M_{\mathrm{ac}}$ we considered. Metal-free accretion can also lead to an improved $\Phi\left({ }^{8} \mathrm{~B}\right)$, but only for smaller $M_{\mathrm{ac}}$ values. Otherwise, for models with $M_{\mathrm{ac}} \gtrsim 0.04 \mathrm{M}_{\odot}$, the predicted $\Phi\left({ }^{8} \mathrm{~B}\right)$ is too large. Because ${ }^{7} \mathrm{Be}$ (panel $\left.b\right)$ is less sensitive to core temperature changes (and less precisely known), significant discrepancies arise only for metal-rich accre- 

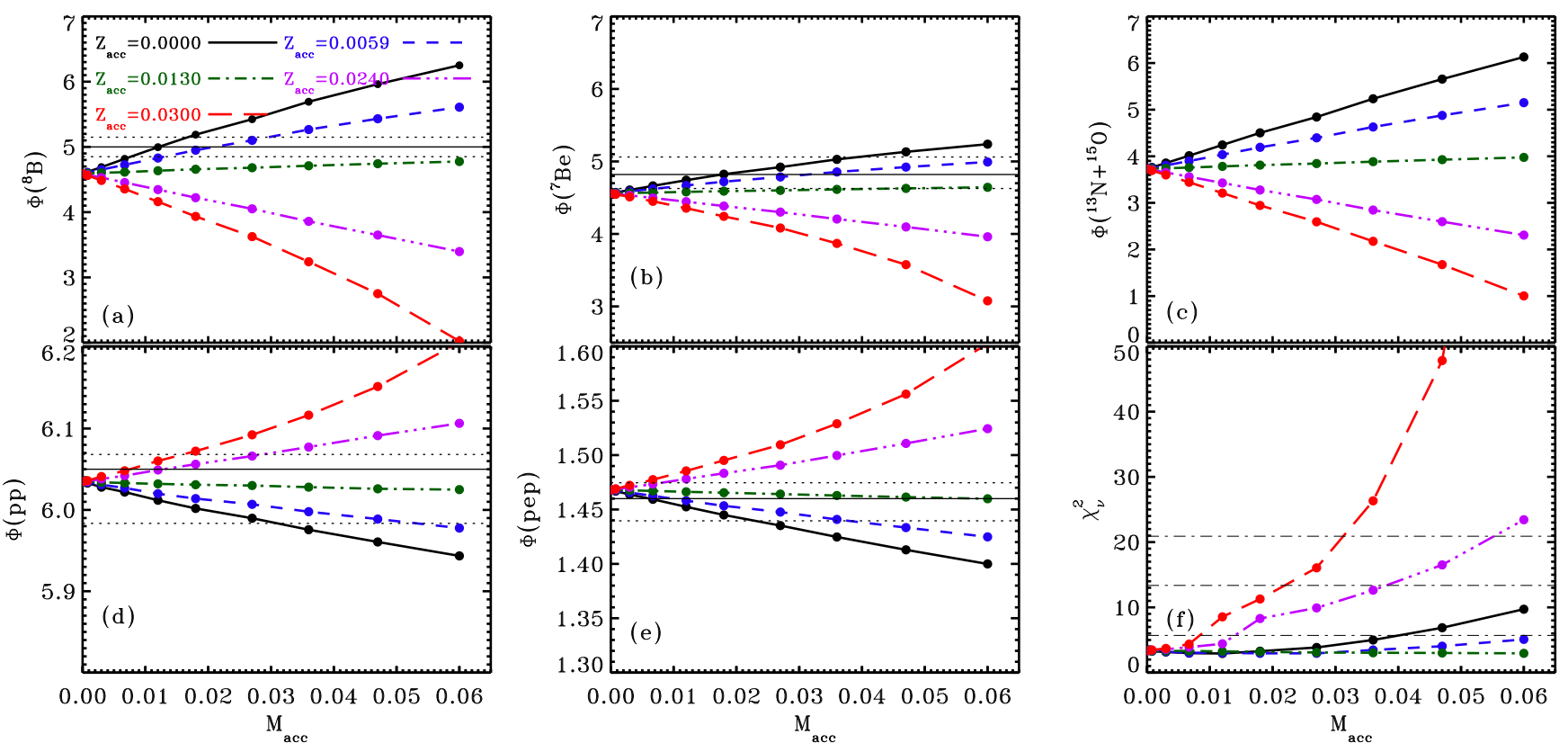

FIG. 10. - Neutrino fluxes for the accreting solar models of Figure 7

tion at large $M_{\mathrm{ac}}$ values. Panel $c$ shows the sensitivity of the combined CN flux to the accretion, but this flux is too poorly constrained experimentally to allow any conclusions to be drawn.

The results for the pp and pep fluxes (panels $d$ and $e$ ), though dependent on the luminosity constraint, strongly suggest that metal-rich accretion can be excluded for $M_{\mathrm{ac}} \gtrsim 0.03 \mathrm{M}_{\odot}$. However, metal-free and metal-poor accretion, over the range of $M_{\mathrm{ac}}$ values we considered, lead to models that are perfectly consistent with the pp and pep solar values. This conclusion is consistent with the one we drew from examining the ${ }^{7}$ Be flux.

The global $\chi^{2}$ in panel $f$ shows that, as in the early accretion case, the metal-free and metal-poor accretion scenarios can slightly improve the overall agreement between the models and the the solar neutrino fluxes. It should be recalled once more, however, that both AGSS09 and GS98 SSMs give excellent agreement with experimentally determined sloar neutrino fluxes (Table 22). The horizontal lines in the plot are the $68.4 \%$, $5 \%$, and $0.1 \%$ probability levels. Comparing the $\chi^{2}$ values of the different $Z_{\mathrm{ac}}$ sequences with results for the individual neutrino fluxes, one finds that the ${ }^{8} \mathrm{~B}$ flux, with its small uncertainty and strong temperature sensitivity, dominates the $\chi^{2}$. For example, although the model with $Z_{\mathrm{ac}}=0.00$ and $M_{\mathrm{ac}}=0.06 \mathrm{M}_{\odot}$ yields nearly perfect agreement with the ${ }^{7} \mathrm{Be}, \mathrm{pp}$, and pep fluxes, a relatively high $\chi^{2}$ is obtained because the model overestimates the ${ }^{8} \mathrm{~B}$ neutrino flux.

\subsection{Late accretion}

\subsubsection{Composition and helioseismic quantities}

In a late accretion model the accretion occurs when the Sun is close to the MS with a well-developed radiative core and consequently a thin convective envelope. The effects of the accretion on the convective envelope are therefore maximal.

The composition and helioseismic properties of the models are summarized in Figure 11. The sequences for $Z_{\mathrm{ac}}=0.024$ and 0.030 terminate at $M_{\mathrm{ac}}=0.02$ and $0.012 \mathrm{M}_{\odot}$ respectively. As is evident from the plot of $Z_{\text {ini }}$ in panel $b$, larger values of $M_{\mathrm{ac}}$ are incompatible with modern Sun's $(Z / X)_{\mathrm{S}}$ : the needed initial metallicity is driven to negative values. Even for smaller, allowed $M_{\mathrm{ac}}$ values, metal-rich accretion leads to very low $Y_{\text {ini }}$ values, well below the SBBN value, as shown in panel $a$.

The helioseismic quantities $R_{\mathrm{CZ}},\langle\delta c / c\rangle$, and $Y_{\mathrm{S}}$ are plotted in panels $d$, $e$, and $f$, respectively. The metal-free and metal-poor accretion models (solid black and blue short dashed lines) yield shallow convective envelopes. Also, for relatively low $M_{\mathrm{ac}}$ values $\left(M_{\mathrm{ac}} \lesssim 0.015 \mathrm{M}_{\odot}\right)$, the overall sound speed profiles, as estimated from $\langle\delta c / c\rangle$ and $Y_{\mathrm{S}}$, are somewhat improved. These models have initial compositions and thus core compositions very similar to that of the GS98 SSM. However, as the models do poorly on $R_{\mathrm{CZ}}$, discrepancies in the sound speed profiles remain. Models with larger $M_{\mathrm{ac}}$ values at low $Z_{\mathrm{ac}}$ fail to reproduce the observed $R_{\mathrm{CZ}}$ and $\langle\delta c / c\rangle$, and also overestimate $Y_{\mathrm{S}}$. We discuss metal-poor accretion further in $\S 5.3$. where we compare our results with previous work.

In contrast to early and intermediate accretion models, sound-speed profiles for late, metal-rich models do not improve, regardless of the $M_{\mathrm{ac}}$ value. The accretion's dramatic effects on surface composition lead to unacceptably low values $Z_{\text {ini }}$ values even for small $M_{\mathrm{ac}}$ values. The resulting low-metallicity cores are not compatible with the radiative-zone sound speed profiles. $Y_{\mathrm{S}}$ is also too low for these models.

\subsubsection{Neutrino fluxes}

Neutrino fluxes are quite sensitive to parameter variations in late accretion models, responding to the large changes in $Z_{\text {ini }}$. The power-law exponents we derive are: $\alpha\left({ }^{7} \mathrm{Be}\right)=0.66, \alpha\left({ }^{8} \mathrm{~B}\right)=1.43, \alpha(\mathrm{CN})=2.08$, $\alpha(\mathrm{pp})=-0.081$, and $\alpha(\mathrm{pep})=-0.18$.

The individual fluxes are given in Figure 12. The agreement between model and observed fluxes deterio- 

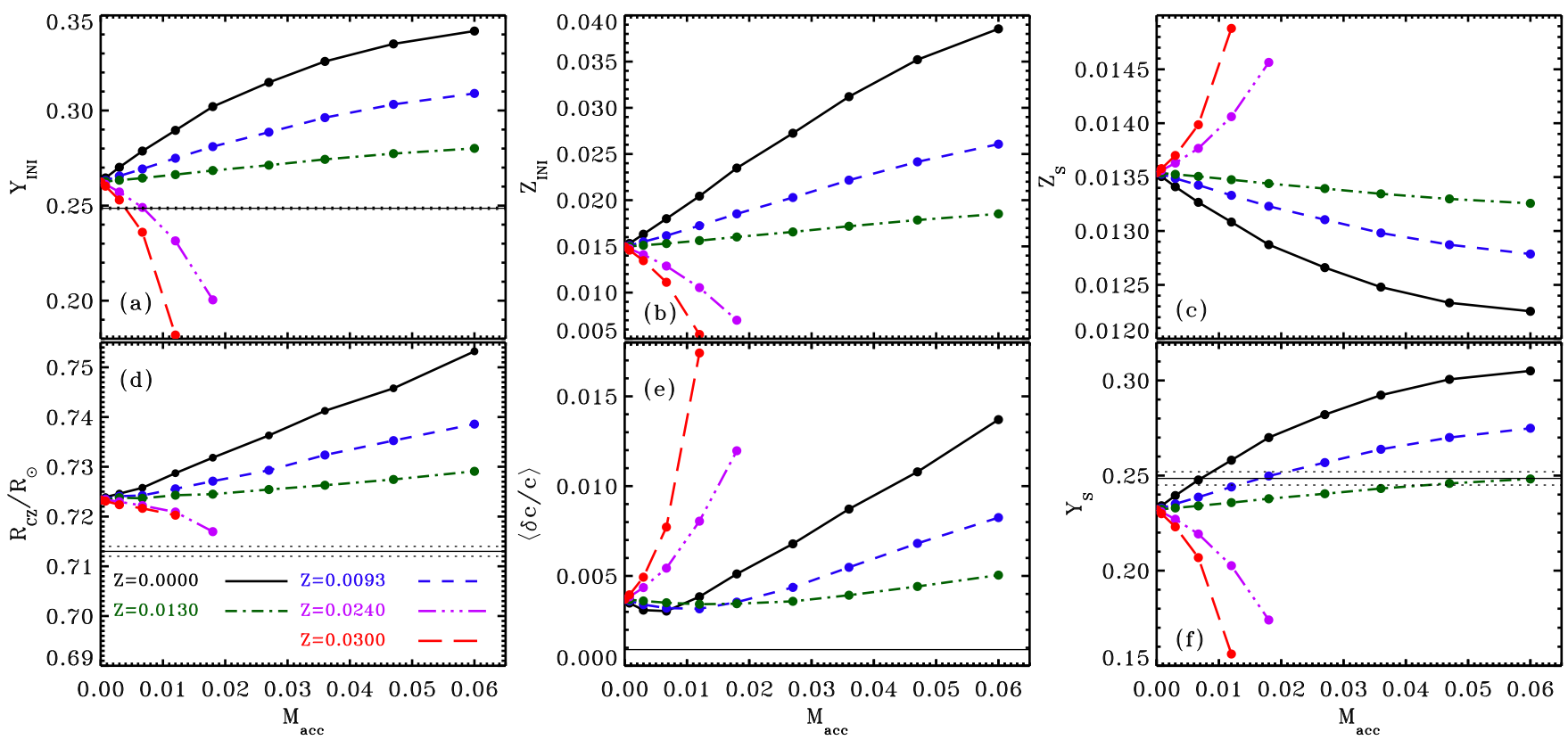

FIG. 11. - Helioseismological quantities and initial composition for accreting solar models with $\tau_{\mathrm{ac}, \mathrm{i}}=30 \mathrm{Myr}$ and $\Delta \tau_{\mathrm{ac}}=10 \mathrm{Myr}(\mathrm{late}$ accretion). Results are shown for five different values of $Z_{\mathrm{ac}}$, labeled in panel $d$.
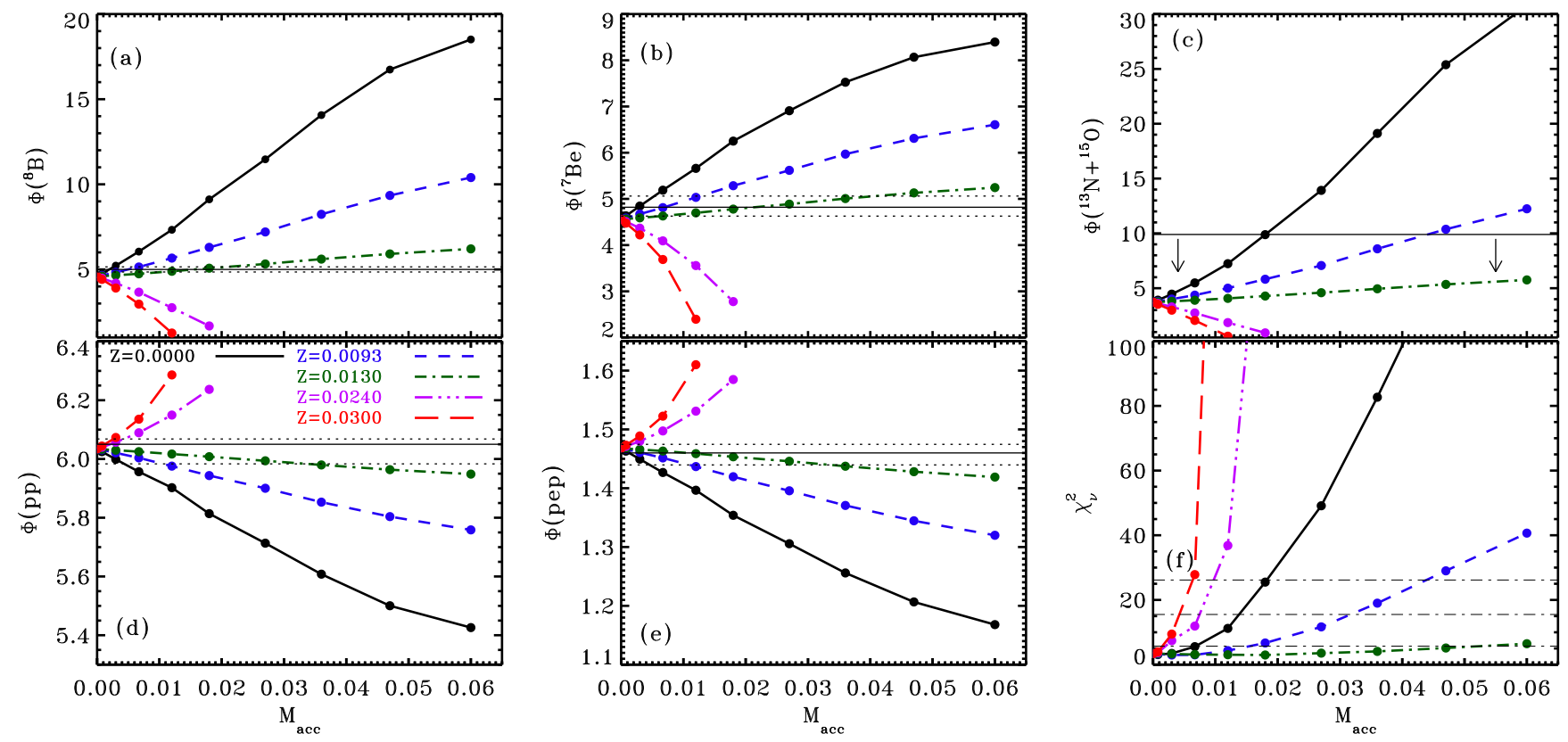

FIG. 12.- Neutrino fluxes for the accreting solar models of Figure 11

rates in both metal-free and metal-rich accretion models. This is mostly driven by the ${ }^{8} \mathrm{~B}$ flux (panel $a$ ), but the other fluxes with relatively small uncertainties $\left({ }^{7} \mathrm{Be}\right.$, $\mathrm{pp}$, and pep) also tend to trend away from observation. Thus the neutrino fluxes limit the accreted composition to near-SSM values, independent of the $M_{\mathrm{ac}}$ value. The slightly metal-poor $Z_{\mathrm{ac}}=0.0093$ sequence is an interesting one, yielding for $M_{\mathrm{ac}} \sim 0.01 \mathrm{M}_{\odot}$ a somewhat improved fit to the observed fluxes. In this model $\langle\delta c / c\rangle$ and $Y_{\mathrm{S}}$ are also somewhat improved, while the convective envelope is only slightly shallower than that of the AGSS09 SSM.

\section{DISCUSSION}

We have investigated whether the solar abundance problem can be solved or alleviated by constructing solar models with different interior and envelope compositions. Rather than postulating an ad hoc modern Sun with an inhomogeneous structure, we instead generate that structure through a physical mechanism, accretion of metal-poor or metal-rich material from the protoplanetary disk. This mechanism is well motivated both because significant accretion is seen in T Tauri stars, which may resemble the early Sun, and because we see clear evidence in our solar system of the the large-scale segre- 
gation of metal that accompanied planetary formation. The chemical processes that concentrated dust and ice at the disk's midplane, and the physical process of planetary formation that scoured those metals from the disk, locking them into the planets, could have combined to create conditions for either metal-poor creation accretion (deposition of metal-depleted gas from the disk's outer surface layers) or metal-rich accretion (proto-planets spiraling into the Sun) after the Sun had developed its radiative core. In this way the composition of the Sun's convective zone could have been altered, becoming chemically distinct from the Sun's radiative core, which would have a composition quite close to that of the solar system's primordial gas.

By exploring accretion as the candidate mechanism responsible for an inhomogeneous modern Sun, we can generate nonstandard models as a function of physical parameters, such as the mass and composition of the accreted gas and the time of accretion. The accretion parameters, and thus the resulting models, are constrained to reproduce the composition of the modern solar surface, taken to be AGSS09 in our models. We can then test these models by comparing their properties against contemporary solar observations done through either helioseismology or solar neutrino spectroscopy. The goal is to determine whether one can account for modern observations that appear to be contradictory in the context of the SSM - the solar abundance problem - through a plausible physical mechanism that could have negated one of the SSM's key assumptions, a homogeneous ZAMS Sun.

The possibility that metal-poor accretion could be responsible for the solar abundance problem has motivated earlier work (Castro et al. 2007; Guzik 2006; Haxton \& Serenelli 2008; Nordlund 2009; Guzik \& Mussack 2010). Our work extends these earlier efforts by exploring a broad range of accretion histories, masses, and compositions, and by testing the resulting models for consistency with modern observations of the Sun's surface and interior. However, there remain unexplored degrees of freedom: we have assumed a constant mass transfer rate, a fixed duration of the accretion, and fixed composition of the accreted material. We discuss our findings and their implications below.

\subsection{Helioseismic quantities}

The most apparent manifestations of the solar abundance problem are the inconsistencies between observations and SSM solar surface helium abundances (too low), convective envelope depths (too shallow), and the solar sound speed profiles, particularly in the upper radiative zone. The main helioseismic results for our updated SSMs can be found in Table 1.

How do the model predictions change if we postulate metal-rich or metal-poor accretion? Let us first consider results for $R_{\mathrm{CZ}}$ and $Y_{\mathrm{S}}$ for the early, intermediate, and late accretion scenarios, shown in panels $d$ and $f$ of Figures 3, 17, and 11] respectively. Qualitatively, metal-poor accretion leads to higher $Y_{\mathrm{S}}$ values and shallower convective envelopes with respect to the SSM. The opposite happens for metal-rich accretion. Therefore, accretion can be invoked to construct models that match the helioseismic values of either $Y_{\mathrm{S}}$ (metal-poor) or $R_{\mathrm{CZ}}$ (metal-
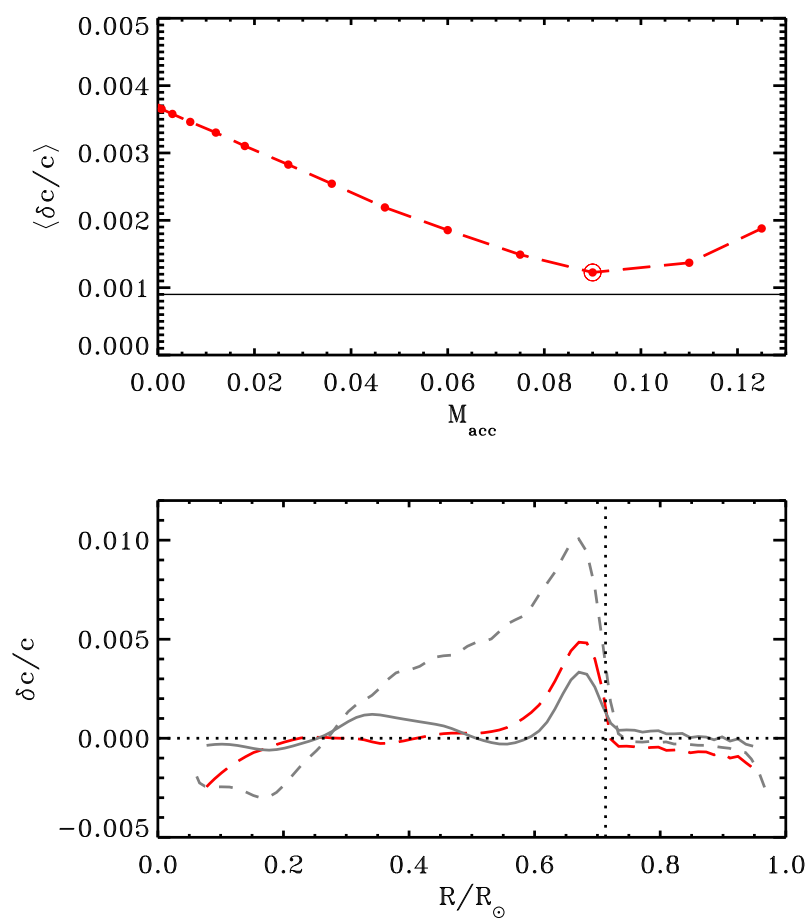

FIG. 13. - Top panel: average rms sound speed for the extended sequence of early accretion models, for $Z_{\mathrm{ac}}=0.030$. Best model, for $M_{\mathrm{ac}}=0.090 \mathrm{M}_{\odot}$, is marked with an open circle. Bottom panel: the red long-dashed line is the sound speed profile for the $M_{\text {ac }}=0.090 \mathrm{M}_{\odot}$ model. The GS98 (grey solid line) and AGSS09 (grey short-dashed) SSM profiles are included for comparison.

rich), but at the expense of enlarging the discrepancy in the other quantity. No model simultaneously improves both $Y_{\mathrm{S}}$ and $R_{\mathrm{CZ}}$.

The discrepancies we observe between solar and model sound speed profiles are correlated with the discrepancies in $R_{\mathrm{CZ}}$. Better agreement in $R_{\mathrm{CZ}}$ leads in general to smaller $\langle\delta c / c\rangle$ values. This is a consequence of $\langle\delta c / c\rangle$ being dominated by the large bump in the sound speed difference right below the convective envelope that is accentuated whenever there is a significant mismatch between the solar and model values for $R_{\mathrm{CZ}}$. The best results for intermediate (late) accretion lead to $\langle\delta c / c\rangle$ being about two (three) times the value for the GS98 SSM. Interestingly, the best results for intermediate accretion are from a metal-rich case (panel $e$ in Figures 7) and for late accretion are from a metal-poor case (panel $e$ in Figures 11). The latter provides an exception to the general rule of correlated $\langle\delta c / c\rangle$ and $R_{\mathrm{CZ}}$, stemming from the sensitivity of $Z_{\text {ini }}$ in late accretion models to the properties of the accreted material. We discuss this further in $\S 5.3$.

For the early accretion scenario, the results displayed in panel $e$ of Figure 3 for metal-rich accretion suggest that further improvement in $\langle\delta c / c\rangle$ might be achieved if $M_{\mathrm{ac}}>0.06 \mathrm{M}_{\odot}$. To test this, we computed models with $Z_{\mathrm{ac}}=0.030$ and $M_{\mathrm{ac}}=0.075,0.090,0.110$, and $0.125 \mathrm{M}_{\odot}$. The results for $\langle\delta c / c\rangle$ are given in Figure 13 (top panel). A minimum is found around $M_{\mathrm{ac}}=0.090 \mathrm{M}_{\odot}$, yielding a value for $\langle\delta c / c\rangle$ close to that of the GS98 SSM (solid black horizontal line). For accretion masses greater than $M_{\mathrm{ac}}=0.090 \mathrm{M}_{\odot},\langle\delta c / c\rangle$ 
begins to rise again. In the bottom panel the sound speed profile for the $M_{\mathrm{ac}}=0.090 \mathrm{M}_{\odot}$ model is shown (red longdashed line). It is similar to that of the GS98 SSM (grey solid line), except close to the Sun's center, where the metallicity and helium abundances in the model are too low. The AGSS09 SSM sound speed profile is also shown. For this model $R_{\mathrm{CZ}}=0.716 \mathrm{R}_{\odot}$, in reasonable agreement with the helioseismic value $0.713 \pm 0.001 R_{\odot}$. However, following the general relation between $R_{\mathrm{CZ}}$ and $Y_{\mathrm{S}}$ previously described, this model has $Y_{\mathrm{S}}=0.225$, further from the helioseismic value, $Y_{\mathrm{S}}=0.2485 \pm 0.0035$, than the AGSS09 SSM value $\left(Y_{\mathrm{S}}=0.2319\right.$, the limit of no accretion). Therefore, larger accretion masses do not offer a global solution to the solar abundance problem.

All calculations presented in Section 4 have been computed assuming a fixed duration for the accretion phase of $\Delta \tau_{\mathrm{ac}}=10 \mathrm{Myr}$. Estimated lifetimes of protoplanetary disks are about a few Myr (Haisch et al. 2001; Williams \& Cieza 2011) so that longer timescales for accretion seem unlikely. On the other hand, shorter timescales are possible, perhaps even favored. We therefore repeated the calculations from $\S 4$ using $\Delta \tau_{\mathrm{ac}}=$ 1 Myr, to test the consequences of shorter accretion times. As one might expect intuitively from an examination of Figure 1, the effect of a reduced $\Delta \tau_{\mathrm{ac}}$ for given $Z_{\mathrm{ac}}, M_{\mathrm{ac}}$, and $\tau_{\mathrm{ac}, \mathrm{i}}$ is analogous to enhancing the dilution factor of the accreted material: that is, for two otherwise equivalent accretion scenarios with the same starting times, the one with the shorter duration will deposit material into a larger mean convection-zone mass during the accretion, and thus that material will experience more dilution. For example, results for $\left(\tau_{\mathrm{ac}, \mathrm{i}}\right.$, $\left.\Delta \tau_{\mathrm{ac}}\right)=(15 \mathrm{Myr}, 1 \mathrm{Myr})$ are intermediate between those for (5 Myr,10 Myr ) and (15 Myr,10 Myr), shown in Figures 3 and 7. respectively. For late accretion, e.g., after the model has settled on the MS, the duration of the accretion phase is irrelevant, provided it is short compared to gravitational settling timescales: at late times, there is little evolution in either the depth or the mass of the convective zone. Thus we conclude that changes in the duration of the accretion will not alter our basic conclusion: no combination of the accretion variables $M_{\mathrm{ac}}, Z_{\mathrm{ac}}$ and $\tau_{\mathrm{ac}, \mathrm{i}}$ will lead to a model in which all helioseismic predictions are improved.

\subsection{Solar neutrinos}

We have compared model solar neutrino fluxes with solar fluxes (column 3 in Table 2). The latter have been derived from a combined analysis of all solar neutrino experiments with the addition of the solar luminosity constraint 3 (Borexino collaboration 2011). The analysis includes the CN-cycle fluxes, although only upper limits are currently available. We find, using the new nuclear reaction rates from SFII and the newest Borexino results for the ${ }^{7} \mathrm{Be}$ flux (Borexino collaboration 2011), that the GS98 and AGSS09 SSMs are both in excellent agreement with solar neutrino data, producing comparable fits. These results are summarized in the last row of Table 2.

Among the four well-determined fluxes, the ${ }^{8} \mathrm{~B}$ neutrinos are the most sensitive to accretion, responding to

\footnotetext{
3 The luminosity constraint equates the solar luminosity to the rate of energy generation in the core, assuming a steady-state Sun.
}

the variations in initial core metallicity that occur because the accretion model is solved under the constraint of a fixed final convective-zone metallicity-to-hydrogen ratio. The ${ }^{8} \mathrm{~B}$ solar flux is also the most certain, now determined to $\sim 3 \%$. Moreover, $\Phi\left({ }^{8} \mathrm{~B}\right)$ provides information complementary to helioseismology because the production region, $R \lesssim 0.1 \mathrm{R}_{\odot}$, is essentially inaccessible to p-mode study. In fact, we find that all models with metal-rich accretion, because of their lower $Z_{\text {ini }}$ values, produce too few ${ }^{8} \mathrm{~B}$ neutrinos. Results are shown in panel $a$ of Figures 6, 10, and 12, This suggests that metal-rich accretion would create a conflict smaller than but reminiscent of the old solar neutrino problem, particularly for the intermediate and late accretion scenarios. In contrast, models with metal-poor accretion, for an appropriate choice of $M_{\mathrm{ac}}$ that depends on the accretion scenario (early, intermediate, or late), can be brought into excellent agreement with the experimental fluxes. The high sensitivity of the ${ }^{8} \mathrm{~B}$ flux to metallicity leads to $M_{\mathrm{ac}}$ values as low as $0.01 \mathrm{M}_{\odot}$ being disfavored for the extreme case of late metal-free accretion.

The ${ }^{7} \mathrm{Be}, \mathrm{pp}$, and pep fluxes, are now also determined to a high level of precision, but are less sensitive to the core conditions (see Figures 6, 10, and 12). With respect to the AGSS09 SSM, a moderate amount of metal-poor accretion can lead to improvements in both the ${ }^{8} \mathrm{~B}$ and ${ }^{7}$ Be fluxes, as discussed below. A slightly more metalrich core seems preferred by these fluxes.

We have done a global analysis of neutrino fluxes, with the results of the $\chi^{2}$ calculations given in panel $f$ of Figures 6, 10, and 12. In the case of early accretion, only the most metal-rich models with massive accretion show significant disagreement with solar data. The neutrino flux responses to accretion are more sensitive in the intermediate and late scenarios, however. Figure 14 gives the resulting $\chi^{2}$ surfaces in the $M_{\mathrm{ac}}-Z_{\mathrm{ac}}$ plane for these scenarios. In each panel the best-fit model is denoted by a red diamond. Solid lines depict the $68.3 \%, 10 \%$, and $1 \%$ probability contours, and dotted lines the contours of probability for the AGSS09 and GS98 SSMs (90\%) (Table 22). In the late accretion case, the top-right corner is empty because no solar models satisfying our requirements can be constructed for such $M_{\mathrm{ac}}-Z_{\text {ac }}$ combinations. These plots define rather clearly the portions of the $M_{\text {ac }}-Z_{\text {ac }}$ parameter space that are consistent with solar neutrino data. For intermediate accretion, all $M_{\mathrm{ac}}$ values considered for $Z_{\text {ac }} \lesssim 0.015$ yield excellent fits, comparable to SSM results. Improvements are possible but minor because, as we have noted before, AGSS09 SSM neutrino flux predictions are in very nice agreement with current solar neutrino data. We conclude that solar neutrino data are primarily of value in ruling out accretion scenarios that give unacceptable fluxes (as opposed to providing positive evidence for accretion scenarios that marginally improve neutrino flux fits). As the accretion becomes more metal-rich, a larger range of high- $M_{\mathrm{ac}}$ accretion scenarios is excluded. In the case of late accretion, large fractions of the $M_{\mathrm{ac}}-Z_{\mathrm{ac}}$ plane are significantly disfavored by solar neutrino data at both the metal-rich and metal-poor extremes. The full $M_{\mathrm{ac}}$ range is allowed only for accretion models with $Z_{\mathrm{ac}} \approx 0.015$ : these are cases close to the SSM.

We close this section on solar neutrinos with a short discussion of the potential role of neutrino fluxes from the 

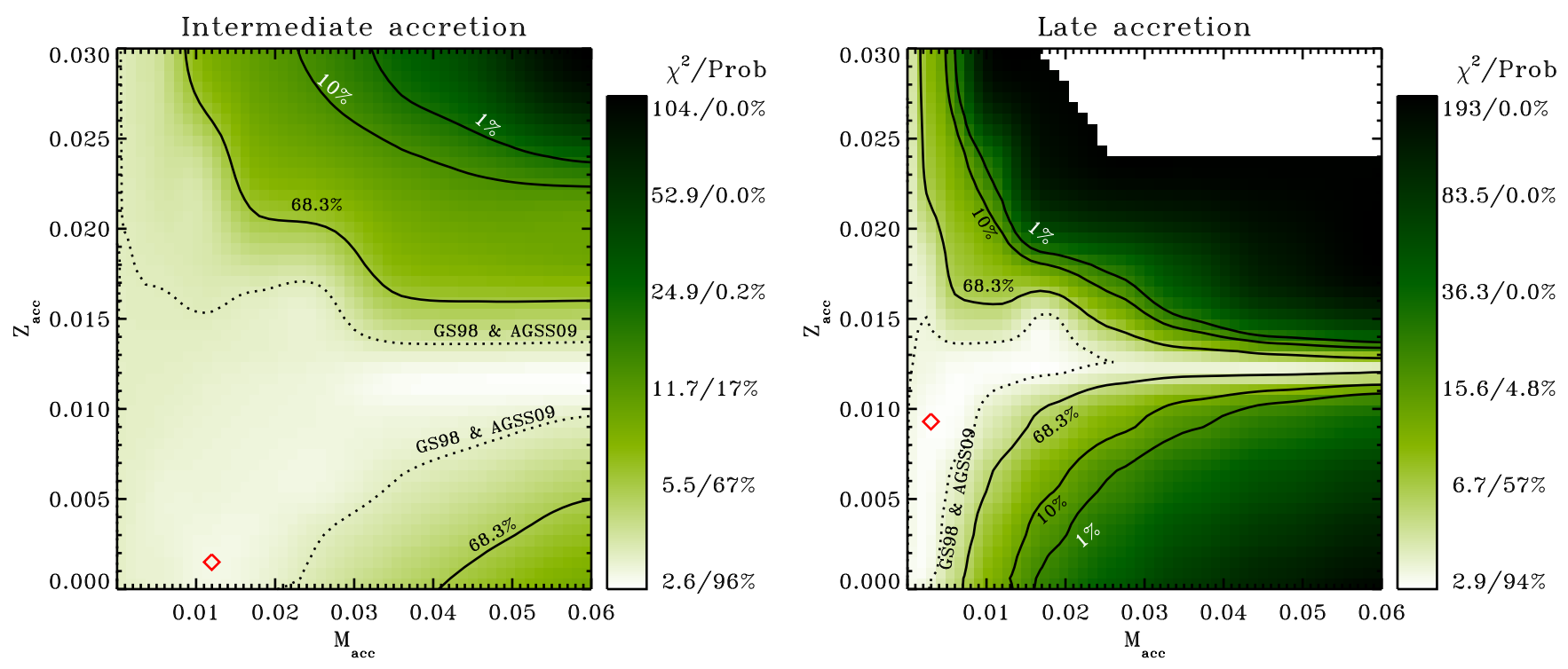

Fig. 14. - Results for the combined $\chi^{2}$ global solar neutrino analysis for the intermediate and late accretion scenarios. Equal probability contours are shown for $1 \%, 10 \%$ and $68.3 \%$. The best fit models are indicated by red diamonds. The fixed- $\chi^{2}$ contours corresponding to the AGSS09 and GS98 SSM fits (Table 2) are overlaid, to indicate regions where accretion models do better or worse than the SSMs.

$\mathrm{CN}$ cycle. These fluxes are very sensitive to metallicity because they respond in two ways: like the ${ }^{8} \mathrm{~B}$ neutrinos, the CN neutrinos respond sharply to environmental effects associated with metallicity - changes in the radiative opacities and the mean molecular weight that alter the temperature of the solar core. But the CN fluxes have an additional linear dependence on the core abundance of the $\mathrm{CN}$ elements, as $\mathrm{C}$ and $\mathrm{N}$ catalyze the hydrogenburning reactions of the CN I cycle. Haxton \& Serenelli (2008) have shown that a future precise measurement of $\Phi\left({ }^{13} \mathrm{~N}\right)$ and $\Phi\left({ }^{15} \mathrm{O}\right)$ could be used to determine the solar core $\mathrm{C}+\mathrm{N}$ abundance to $\sim 10 \%$, including all solar model and neutrino parameter uncertainties (see also Serenelli et al. 2011). A direct determination of the central $\mathrm{C}+\mathrm{N}$ abundance would be very valuable, as a comparison to surface abundances would constitute a direct test of the SSM hypothesis of a homogeneous ZAMS Sun. In our models we find that the central-to-surface metallicity ratio, normalized to the SSM value to account for the effects of microscopic diffusion, deviates from unity by more than $10 \%$ in many of our models, particularly for the intermediate and late accretion scenarios, as illustrated in Figure 15. Thus a high-quality CN flux measurement could help rule out a large portion of the potential parameter space of accretion models, making the measurement an important diagnostics of the early Sun and its proto-planetary disk. Of course, this is in the context of the assumptions we have made in our accretion scenarios, which include a uniform rate of accretion of material with a metallicity related to AGSS09 by a simple scale factor, onto a proto-Sun with a metallicity that is also scaled to AGSS09. As disk condensation temperatures vary from element to element, there is ample motivation for relaxing these assumptions. (More general scenarios in which such assumptions are reconsidered will be discussed elsewhere.) Despite this caveat, we believe that the ${ }^{13} \mathrm{~N}$ and ${ }^{15} \mathrm{O}$ neutrino fluxes are potentially very useful as probes of solar core metallicity and, therefore, potentially of the chemistry of the proto-solar disk. This chemistry sets the conditions for the formation of the solar system's diverse planets, and, as we have speculated, may have also altered the composition of the solar surface. Given that there exist very few quantitative probes of the processes that governed solar system formation, it is important to pursue any experiment that can directly constrain phenomena like accretion during the formation period.

\subsection{Comparison with previous works}

Guzik (2006); Guzik \& Mussack (2010) and Castro et al. (2007) have studied accretion of metal-poor material as a possible solution to the solar abundance problem. The authors constructed solar models in which the interior metallicity is high (around the GS98 value) and the envelope metallicity is low, consistent with Asplund et al. (2005). Guzik (2006) assumes that a mass of $0.02 \mathrm{M}_{\odot}$ is accreted during a period of $36 \mathrm{Myr}$, onto a ZAMS stellar model of $0.98 \mathrm{M}_{\odot}$. Castro et al. (2007) takes a simplified approach in which the metallicity of the convective envelope is simply scaled down by a given factor (0.5) when the solar model is 74 Myr old. In both treatments the accretion is assumed to occur after the contraction phase to the MS is over, when the convective envelope is thin and very close to its present-day extension. In this aspect, their models resemble our late accretion scenario.

In terms of $Z_{\text {ini, two of our late-accretion models }}$ closely resemble the cases studied Guzik (2006) and Castro et al. (2007). These are our models with $Z_{\text {ac }}=$ 0.0093 and with $M_{\mathrm{ac}}=0.013$ and $0.018 \mathrm{M}_{\odot}$, respectively (see Figures 11 and 12). The sound speed profiles of these two models, shown in Figure [16, match very well that of the GS98 SSM below $R / R_{\odot}=0.5$, but the bump below the convective zone remains quite prominent. Therefore $\langle\delta c / c\rangle$ shows only modest improvement over the AGSS09 SSM value. These results correspond to Figure 12 of Guzik (2006) and to Figure 1 of 


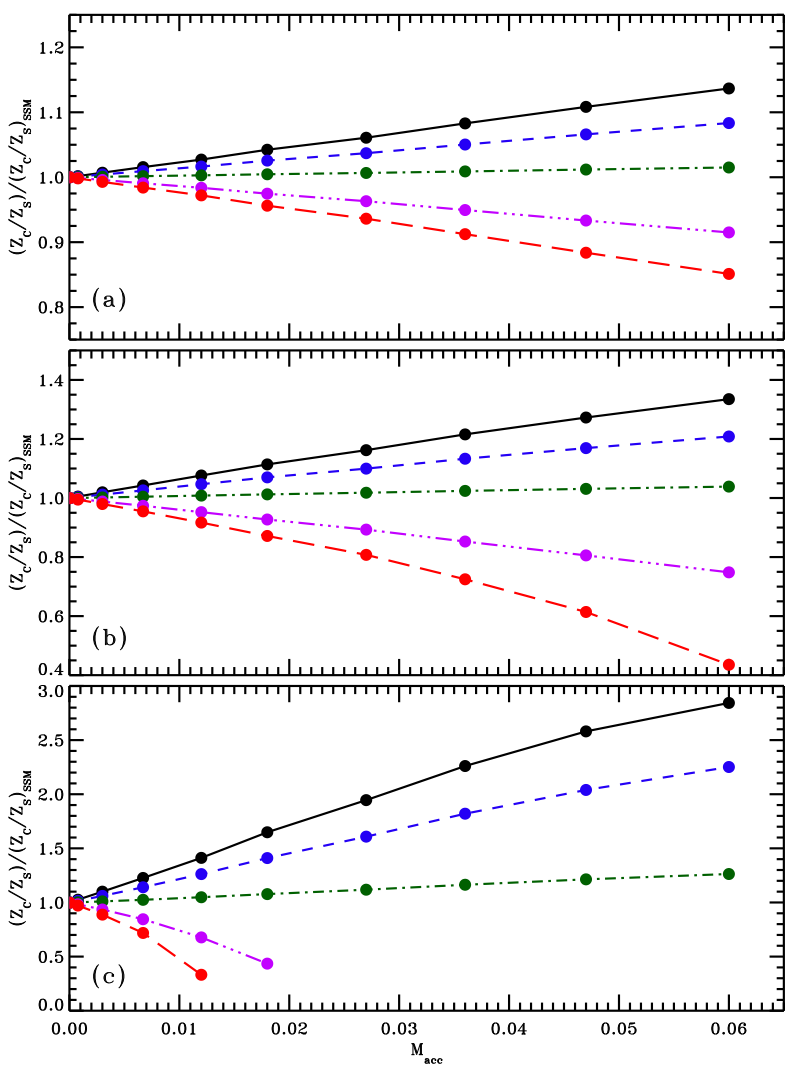

FIG. 15.- Ratio of the central to surface metallicity in selected models. Panels $a, b$, and $c$ refer to early, intermediate and late accretion scenarios respectively. Metallicity sequences are shown for $Z_{\mathrm{ac}}=0,0.0059,0.0130,0.0240,0.0300$ (top to bottom in each panel).

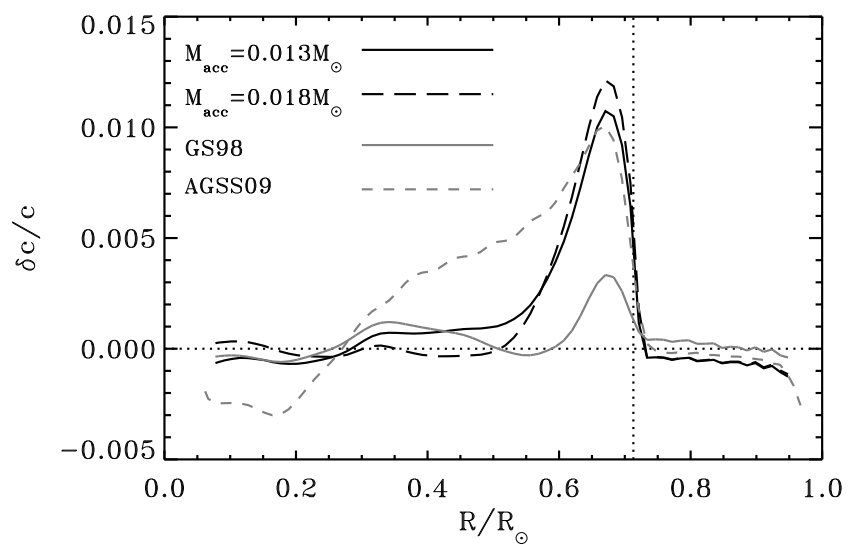

FIG. 16. - Sound speed profiles for the models with $M_{\mathrm{ac}}=0.013$ (black solid line) and $0.018 \mathrm{M}_{\odot}$ (red dashed-dotted line), $Z_{\mathrm{ac}}=$ $0.0093, \tau_{\mathrm{ac}, \mathrm{i}}=30 \mathrm{Myr}$, and $\Delta \tau_{\mathrm{ac}}=10 \mathrm{Myr}$. GS98 (grey solid dashed) and AGSS09 (grey dashed line) standard solar models are shown for comparison.

Castro et al. (2007). Our models also yield $R_{\mathrm{CZ}}=0.725$ and $0.727 \mathrm{R}_{\odot}$, respectively, so the agreement with helioseismology in this quantity is slightly worse than for the AGSS09 SSM $\left(0.723 \mathrm{R}_{\odot}\right)$. These observations are in qualitative agreement with Castro et al. (2007), who also find that the convective zone gets shallower $\left(0.732 \mathrm{R}_{\odot}\right.$ instead of $\left.0.730 \mathrm{R}_{\odot}\right)$ in the model with accretion. Guzik
(2006), on the other hand, finds improvement in $R_{\mathrm{CZ}}$ with respect to the model with no accretion. The reason for the qualitatively different behavior is not clear, but plausibly could be connected to the assumed effects of the accretion on the convective zone's hydrogen and helium abundances. Finally, our models give $Y_{\mathrm{S}}=0.244$ and 0.250 , in good agreement with the helioseismic value, whereas Guzik (2006) and Castro et al. (2007) find $Y_{\mathrm{S}}=0.240$, marginally compatible with the seismic result. The small numerical discrepancies may be due to the use of somewhat different solar compositions in the three sets of calculations. Yet, overall the resulting models are in reasonable agreement, given the differences in the manner accretion was implemented in the three studies.

\section{SUMMARY AND CONCLUSIONS}

In this study we have explored the possibility that an accretion phase, occurring after the Sun developed a radiative core, could resolve, or at least mitigate, the solar abundance problem. The motivation for considering this possibility is the observation that large-scale metal segregation occurred in the proto-planetary disk, connected with the formation of the planets. Extending previous studies, we considered both metal-poor and metal-rich accretion and also allowed accretion to occur at different evolutionary stages of the young Sun. We also considered the constraints on models from both helioseismology and neutrino measurements, while earlier studies focused on the former.

We began by updating the SSM, adopting the nuclear reaction rates from the very recent evaluation of SFII. The new SSMs for ASSS09 and GS98 abundances then were used as benchmark calculations against which the effects of accretion could be measured. The new models differ only modestly from others recently calculated (Serenelli et al. 2009). The new solar models with the lower metallicity AGSS09 composition remain in conflict with helioseismology. The newly recommended nuclear fusion rates do impact predicted SSM solar neutrino fluxes. The higher metallicity GS98 SSM remains in excellent agreement with solar neutrino data, but now the lower metallicity AGSS09 SSM shows an equivalent level of agreement. This also partly the result of the most recent ${ }^{7} \mathrm{Be}$ flux measurement by Borexino (Borexino collaboration 2011). Thus solar neutrino spectroscopy (and the associated laboratory astrophysics) has not yet reach the level of precision necessary to distinguish between the AGSS09 and GS98 SSMs.

The main conclusion we draw from our study of accretion is that, for the class of models we explored, no scenario was found to provide a satisfactory solution of the solar abundance problem. Here "satisfactory solution" is defined as a model with AGSS09 surface abundances that agrees with the known helioseismic properties as well as the GS98 SSM. We find that metal-rich accretion can bring the depth of the convective envelope into agreement with the seismic value, but that the resulting surface helium abundances in such models are always too low - in fact, in some of these models the initial helium abundance falls below the primordial SBBN value. Conversely, some metal-poor accretion models bring surface helium abundance into a very nice agreement with the solar value, but at the cost of a convective envelope that 
is even shallower than that of the AGSS09 SSM.

Problems in the sound speed profile are generally strongly correlated with the problems in the depth of the convective envelope. The better (worse) the latter, the better (worse) the former. An exception to this general rule is found for late accretion models in which about $0.015 \mathrm{M}_{\odot}$ of metal-poor material is accreted. In this case, though the model's convective envelope is too shallow, there is some improvement in the sound speed profile. However, a significant bump in the sound speed difference (model vs. heliosesimology) remains in the region right below the convective envelope. These models have surface helium abundances and neutrino fluxes that agree with solar values.

Current solar neutrino data constrain solar core properties. Our global comparison of model and solar fluxes rules out both metal-poor and metal-rich accretion for $M_{\mathrm{ac}} \gtrsim 0.01 \mathrm{M}_{\odot}$ in the late accretion scenario.

The results of our accretion study provide further motivation for the proposal to exploit future measurements of $\mathrm{CN}$ neutrinos as a test of the $\mathrm{C}+\mathrm{N}$ abundance of the solar core (Haxton \& Serenelli 2008). We find that the ratio between the surface and central solar metallicity in accretion models deviates from unity (gravitational settling taken into account) by more than $10 \%$ for most accretion scenarios. Such a measurement of the solar core bulk abundance of $\mathrm{C}+\mathrm{N}$ does not directly constrain other metals and thus the overall metallicity: despite the simplifying assumptions made in our accretion model, there is no a priori reason to believe that the composition of accreted gas is related to the primordial gas by a simple scale factor. Still, any quantitative abundance determination in the solar interior would be a major step forward.
In the past, accretion of metal-rich material was discussed as a possible solution to the solar neutrino problem (Winnick et al. 2002). More recently, a new lowmetallicity solar composition has generated concern that the Sun's surface might have been altered in an episode of metal-poor accretion (Guzik 2006; Castro et al. 2007). Additional motivations for continuing the study of accretion onto the early Sun can be found in suggestions that the mechanism generating metal-poor or metal-rich accretion might be connected to the dynamics of the protoplanetary disk. The possibility of such a connection has been raised in several papers: in the observation that solar abundance problem involves a deficit of convectivezone metal comparable to the excess of metal sequestered in the planets (Haxton \& Serenelli 2008); in recent models of the assembly of low mass stars (Baraffe \& Chabrier 2010); and in claims that the Sun has an abundance pattern of metals that correlates with metal condensation temperatures, suggesting a connection with disk chemistry and the formation of rocky planets (Meléndez et al. 2009). Work on accretion scenarios motivated by these observations is ongoing and will be reported elsewhere.

Aldo Serenelli is partially supported by the European Union International Reintegration Grant PIRGGA-2009-247732, the MICINN grant AYA08-1839/ESP, by the ESF EUROCORES Program EuroGENESIS (MICINN grant EUI2009-04170), by SGR grants of the Generalitat de Catalunya and by the EU-FEDER funds. W. C. Haxton is supported in part by the U.S. Department of Energy, including under de-sc00046543 at Berkeley. CPG is supported by the grants FPA-2007-60323 and PROMETEO/2009/116. We thank Maria Bergemann for useful discussions and her careful reading of the manuscript.

\section{REFERENCES}

Adelberger, E. G., et al. 1998, Rev. Mod. Phys., 70, 1265 Adelberger, E. G., et al. 2010, arXiv:1004.2318 and to be published, Rev. Mod. Phys.

Aharmim, B., et al. 2010, Phys. Rev. C, 81, 055504

Ahmad, Q. R., et al. 2002, Phys. Rev. Lett., 89, 011301

Allende Prieto, C., Lambert, D. L., \& Asplund, M. 2001, ApJ, 556, L63

Ando, S., et al. 2008, Phys. Lett. B, 668, 187

Angulo, C., et al. 1999, Nucl. Phys. A, 656, 3

Antia, H. M., \& Basu, S. 2005, ApJ, 620, L129

Antia, H. M., \& Basu, S. 2006, ApJ, 644, 1292

Arpesella, C. et al. 2008, Phys. Rev. Lett., 101, 091302

Asplund, M., Grevesse, N., Sauval, A. J., Allende Prieto, C., \& Kiselman, D. 2004, A\&A, 417, 751

Asplund, M., Grevesse, N., \& Sauval, A. J. 2005, Cosmic Abundances as Records of Stellar Evolution and Nucleosynthesis, 336, 25

Asplund, M., Grevesse, N., Sauval, A. J., \& Scott, P. 2009, ARA\&A, 47, 481

Asplund, M., Nordlund, A., Trampedach, R., Allende Prieto, C., \& Stein, R. F. 2000, A\&A, 359, 729

Baby, L. T., et al. 2003a, Phys. Rev. Lett., 90, 022501

Baby, L. T., et al. 2003b, Phys. Rev. C, 67, 065805;

Bahcall, J. N., Basu, S., Pinsonneault, M., \& Serenelli, A. M. 2005, ApJ, 618, 1049

Bahcall, J. N., Basu, S., \& Serenelli, A. M. 2005, ApJ, 631, 1281

Bahcall, J. N., \& Peña-Garay, C. 2003, Journal of High Energy Physics, 11, 4

Bahcall, J. N., Pinsonneault, M. H., \& Basu, S. 2001, ApJ, 555, 990

Bahcall, J. N., Serenelli, A. M., \& Basu, S. 2005, ApJ, 621, L85
Bahcall, J. N., Serenelli, A. M., \& Basu, S. 2006, ApJS, 165, 400 Bahcall, J. N., Serenelli, A. M., \& Pinsonneault, M. 2004, ApJ, 614,464

Bahcall, J. N. \& Ulmer, A. 1996, Phys. Rev. D, 53, 4202

Baraffe, I., Chabrier, G., \& Gallardo, J. 2009, ApJ, 702, L27

Baraffe, I., \& Chabrier, G. 2010, A\&A, 521, A44

Basu, S., \& Antia, H. M. 2004, ApJ, 606, L85

Basu, S., \& Antia, H. M. 1997, MNRAS, 287, 189

Bellini, G., et al. 2010, Phys. Rev. D, 82, 033006

Bemmerer, D., et al. 2006a, Phys. Rev. Lett., 97, 122502

Bemmerer, D., et al. 2006b, Nucl. Phys. A, 779, 297

Bemmerer, D., et al. 2009, J. Phys. G, 36, 045202

Bergemann, M., Pickering, J. C., \& Gehren, T. 2010, MNRAS, 401, 1334

Bonetti, R., et al. 1999, Phys. Rev. Lett., 82, 5205

Borexino collaboration 2011, in preparation

Brown, T. A. D., et al. 2007, Phys. Rev. C, 76, 055801

Butler, M. \& Chen, J.-W. 2001, Phys. Lett. B, 520, 87

Caffau, E., Ludwig, H.-G., Steffen, M., Freytag, B., \& Bonifacio, P. 2010, Sol. Phys., 66

Carlson, J., et al. 1991, Phys. Rev. C, 44, 619

Castro, M., Vauclair, S., \& Richard, O. 2007, A\&A, 463, 755

Christensen-Dalsgaard, J., et al. 1996, Science, 272, 1286

Christensen-Dalsgaard, J., di Mauro, M. P., Houdek, G., \& Pijpers, F. 2009, A\&A, 494, 205

Confortola, F., et al. 2007, Phys. Rev. C, 75, 065803

Costantini, H., et al. 2008, Nucl. Phys. A, 814, 144

Delahaye, F., \& Pinsonneault, M. H. 2006, ApJ, 649, 529

Delahaye, F., Pinsonneault, M. H., Pinsonneault, L., \& Zeippen, C. J. 2010, arXiv:1005.0423

Desch, S. J. 2007, ApJ, 671, 878 
di Leva, A., et al. 2009, Phys. Rev. Lett., 102, 232502

Fedele, D., van den Ancker, M. E., Henning, T., Jayawardhana, R., \& Oliveira, J. M. 2010, A\&A, 510, A72

Filippone, B. W., Elwyn, A. J., Davids, C. N., \& Koetke, D. D. 1983a, Phys. Rev. Let., 50, 412

Filippone, B. W., Elwyn, A. J., Davids, C. N., \& Koetke, D. D. 1983b, Phys. Rev. C, 28, 2222

Formicola, A., et al. 2004, Phys. Lett. B, 591, 61

Freytag, B., Steffen, M., \& Dorch, B. 2002, Astronomische Nachrichten, 323, 213

González-García, M. C., Maltoni, M., \& Salvado, J. 2010, Journal of High Energy Physics, 5, 72

Grevesse, N., \& Noels, A. 1993, Origin and Evolution of the Elements, 15

Grevesse, N., \& Sauval, A. J. 1998, Space Sci. Rev., 85, 161

Guzik, J. A. 2006, in ESA Special Publication, Vol. 624, Proceedings of SOHO 18/GONG 2006/HELAS I, Beyond the spherical Sun

Guzik, J. A., \& Mussack, K. 2010, ApJ, 713, 1108

Gyurky, G., et al. 2007, Phys. Rev. C, 75, 035805

Haisch, K. E., Jr., Lada, E. A., \& Lada, C. J. 2001, ApJ, 553, L153

Hammache, F., et al. 1998, Phys. Rev. Lett., 80, 928;

Hammache, F., et al. 2001, Phys. Rev. Let., 86, 3985

Haxton, W. C., \& Serenelli, A. M. 2008, ApJ, 687, 678

Hayashi, C. 1981, Prog. Theor. Phys. Suppl., 70, 35

Hebbard, D. F. 1960, Nucl. Phys., 15, 289

Imbriani, G., et al. 2005, Eur. Phys. J. A, 25, 455

Junghans, A. R., et al. 2002, Phys. Rev. Lett., 88, 041101

Junghans, A. R., et al. 2003, Phys. Rev. C, 68, 065803

Junghans, A. R., et al. 2010, Phys. Rev. C, 81, 012801(R)

Kong, X. \& Ravendahl, F. 2001, Phys. Rev. C, 64, 044002

Kurylov, A. M., Ramsey-Musolf, M. J., \& Vogel, P. 2003, Phys. Rev. C, 67, 035502

Larson, D., et al. 2010, ArXiv e-prints

Lemut, A., et al. 2006, Phys. Lett. B, 634, 483
Marcucci, L. E., et al. 2000, Phys. Rev. C, 63, 015801

Marta, M., et al. 2008, Phys. Rev. C, 78, 022802(R)

Meléndez, J., Asplund, M., Gustafsson, B., \& Yong, D. 2009, ApJ, 704, L66

Montalbán, J., Miglio, A., Noels, A., Grevesse, N., \& di Mauro, M. P. 2004, SOHO 14 Helio- and Asteroseismology: Towards a Golden Future, 559, 574

Nordlund, A. 2009, arXiv:0908.3479

Park, T. S., et al. 2003, Phys. Rev. C67, 055206

Peña-Garay, C., \& Serenelli, A. 2008, arXiv:0811.2424

Przybilla, N., Nieva, M.-F., \& Butler, K. 2008, ApJ, 688, L103

Rolfs, C., \& Rodney, W. S. 1974, Nucl. Phys. A, 235, 340

Runkle, R. C., et al. 2005, Phys. Rev. Lett., 94, 082503

Schiavilla, R., et al. 1998, Phys. Rev. C, 58, 1263

Schlattl, H., Weiss, A., \& Raffelt, G. 1999, Astroparticle Phys., 10,353

Serenelli, A. M. \& Basu, S. 2010, ApJ, 719, 865

Serenelli, A. M., Basu, S., Ferguson, J. W., \& Asplund, M. 2009, ApJ, 705, L123

Serenelli, A. M., Peña-Garay, C., \& Haxton, W. C. 2011, in preparation

Singh, B. S., Hass, M., Nir-El, Y., \& Haquin, G. 2004, Phys. Rev. Lett., 93, 262503

Stein, R. F., \& Nordlund, A. 1998, ApJ, 499, 914

Strieder, F., et al. 2001, Nucl. Phys. A, 696, 219

Turck-Chièze, S., et al. 2001, ApJ, 555, L69

Wedemeyer, S., Freytag, B., Steffen, M., Ludwig, H.-G., \& Holweger, H. 2004, A\&A, 414, 1121

Weidenschilling, S. J. 1977, Ap\&SS, 51, 153

Weiss, A. \& Schlattl, H. 2008, Ap\&SS, 316, 99

Williams, J. P., \& Cieza, L. A. 2011, arXiv:1103.0556

Winnick, R. A., Demarque, P., Basu, S., \& Guenther, D. B. 2002, ApJ, 576, 1075

Wuchterl, G., \& Klessen, R. S. 2001, ApJ, 560, L185 